\title{
RESEARCH OF A MATHEMATICAL MODEL OF LOW-CONCENTRATED AQUEOUS POLYMER SOLUTIONS
}

\author{
MIKHAIL V. TURBIN
}

Received 12 March 2005; Accepted 10 July 2005

The initial-boundary value problem for the mathematical model of low-concentrated aqueous polymer solutions is considered. For this initial-boundary value problem a concept of a weak solution is introduced and the existence theorem for such solutions is proved.

Copyright (c) 2006 Mikhail V. Turbin. This is an open access article distributed under the Creative Commons Attribution License, which permits unrestricted use, distribution, and reproduction in any medium, provided the original work is properly cited.

\section{Introduction}

The study of fluid motion is a source of a large number of mathematical problems. Motion of an incompressible fluid in a bounded domain $\Omega \subset \mathbf{R}^{n}$ on a time interval $[0, T]$, $(T<\infty)$ is described by the following system of equations in Cauchy's form [3]:

$$
\begin{gathered}
\frac{\partial v}{\partial t}+v_{i} \frac{\partial v}{\partial x_{i}}+\operatorname{grad} p=\operatorname{Div} \sigma+f, \quad(x, t) \in \Omega \times[0, T], \\
\operatorname{div} v=0, \quad(x, t) \in \Omega \times[0, T] .
\end{gathered}
$$

Hereinafter Einstein's summation convention is supposed. In the given system $v(x, t)$ is the velocity vector of the particle at a point $x$ at an instant $t ; p=p(x, t)$ is the pressure of the fluid at a point $x$ at an instant $t ; f=f(x, t)$ is the vector of body forces acting on the fluid; $\sigma=\left(\sigma_{i j}(x)\right)$ is the deviator of the stress tensor. By Div $\sigma$ we denote the vector of divergences of columns of the matrix $\sigma$.

The system of (1.1) describes formally the motion of all kinds of fluids. However the number of unknowns in this system is greater than the number of equations. To complete the given system one usually uses various relations between the deviator of the stress tensor $\sigma$ and the strain rate tensor $\mathscr{E}=\left(\mathscr{E}_{i j}\right)$,

$$
\mathscr{E}_{i j}=\mathscr{E}_{i j}(v)=\frac{1}{2}\left(\frac{\partial v_{i}}{\partial x_{j}}+\frac{\partial v_{j}}{\partial x_{i}}\right)
$$

Hindawi Publishing Corporation

Abstract and Applied Analysis

Volume 2006, Article ID 12497, Pages 1-27

DOI 10.1155/AAA/2006/12497 
Such relations are called constitutive laws. A constitutive law is a hypothesis which should be verified for concrete fluids by experimental data.

The most known constitutive law for viscous incompressible liquid is Newton's constitutive law

$$
\sigma=2 \nu \mathscr{E}
$$

Here $v$ is the viscosity of the fluid. This model describes the motion of the majority of viscous incompressible fluids appearing in practice at moderate velocities. However this model does not describe the motion of the fluids possessing relaxational properties. These properties consist in the following. After a change of the exterior conditions the equilibrium state in a fluid does not appear at once but after a while. This while is determined by the processes of interior reorganization. These processes may have various nature (magnetic, thermal, etc.). The fluid may have some spectrum of relaxation times corresponding to these processes.

In papers $[16,18]$ it has been noted, that the account of relaxational properties of a fluid is the account of time connections between the deviator of the stress tensor $\sigma$ and the strain rate tensor $\mathscr{E}$. The presence of this connection expresses itself in losses of the mechanical energy of a flow, additional to the dissipative losses owing to viscosity. These additional losses of the mechanical energy of the flow are to be taken into account in the general case of motion of a fluid.

The following relation was suggested in paper [16] as a one taking into account the mentioned effects:

$$
\sigma=2 \nu\left(\mathscr{E}+\varkappa \nu^{-1} \frac{d \mathscr{E}}{d t}\right), \quad \varkappa, \nu>0
$$

Here $v$ is the kinematic coefficient of viscosity, and $\varkappa$ is the retardation time. Sometimes the coefficient $\varkappa$ is also called the time of strain relaxation. This model describes the motion of viscous non-Newtonian fluid which requires time to begin a motion after an action of a force suddenly applied.

Substituting $\sigma$ from the constitutive law (1.4) into the system of equations of incompressible liquid motion in Cauchy's form (1.1) we obtain the following system of equations:

$$
\begin{gathered}
\frac{\partial v}{\partial t}-v \Delta v+v_{i} \frac{\partial v}{\partial x_{i}}-\varkappa \frac{\partial \Delta v}{\partial t}-\varkappa \operatorname{Div}\left(v_{k} \frac{\partial \mathscr{E}(v)}{\partial x_{k}}\right)+\operatorname{grad} p=f, \quad x \in \Omega, t \in[0, T] \\
\operatorname{div} v=0, \quad x \in \Omega, t \in[0, T] .
\end{gathered}
$$

The given system of equations differs from the Navier-Stokes system of equations by presence of the additional term $\varkappa \operatorname{Div}(d \mathscr{E} / d t)$. This term describes relaxational properties of a fluid. In the case of very weak relaxational properties of the fluid (for $\varkappa$ close to zero), and also in the case when the motion of the liquid has steady-state character (the total derivative of the strain rate tensor with respect to time is equal to zero) this additional term vanishes. In this case the system of (1.5), (1.6) coincides with the system of Navier-Stokes 
equations. But in the case of turbulent behavior of the fluid and on unsteady laminar behavior of the fluid this additional term is nonzero and must play a significant role. Just so low-concentrated aqueous solutions of polymers behave and it has been confirmed by experimental researches of polyethylenoxide and polyacrylamide aqueous solutions [2] and polyacrylamide and guar gum aqueous solutions [1]. Therefore the given model has also received the title of the mathematical model of motion of low-concentrated aqueous polymer solutions.

Note also that the constitutive law (1.4) is a special case of Noll's constitutive law [9]:

$$
\sigma_{i j}=\varphi\left(I_{2}\right) \mathscr{E}_{i j}+\psi\left(I_{2}\right) \mathscr{E}_{i k} \mathscr{E}_{k j}+\psi_{1}\left(I_{2}\right)\left(\frac{\partial \mathscr{E}}{\partial t}+v_{k} \frac{\partial \mathscr{E}_{i j}}{\partial x_{k}}-\left[\frac{\partial v_{i}}{\partial x_{m}} \mathscr{E}_{m j}+\frac{\partial v_{j}}{\partial x_{m}} \mathscr{E}_{i m}\right]\right) .
$$

Here $\varphi\left(I_{2}\right), \psi\left(I_{2}\right), \psi_{1}\left(I_{2}\right)$ are material functions depending on the invariant $I_{2}=\mathscr{E}_{i k} \mathscr{E}_{k i}$. Noll's model of fluid motion is a very general model for which the stress tensor at moment $t$ is determined by nonlinear symmetrical tensor functional. This functional is defined on the set of tensor functions determining history of deformation of a medium at all times preceding the considered instant $t$.

In this paper we consider the system of (1.5), (1.6) in a bounded domain $\Omega \subset \mathbf{R}^{n}$, $n=2,3$ with locally-Lipschitz boundary on a time interval $[0, T],(T<\infty)$. For $(1.5)$, (1.6) we study the initial-boundary value problem with the initial condition

$$
v(x, 0)=a(x), \quad x \in \Omega,
$$

and the boundary condition

$$
\left.v\right|_{\partial \Omega \times[0, T]}=0 .
$$

We do not know papers in which problem (1.5)-(1.9) would have been investigated. Since this problem is very complicated for the research. In works $[11,16]$ it was suggested the following simplification:

$$
\begin{gathered}
\frac{\partial v}{\partial t}-v \Delta v+v_{i} \frac{\partial v}{\partial x_{i}}-\varkappa\left(\frac{\partial \Delta v}{\partial t}+v_{k} \frac{\partial \Delta v}{\partial x_{k}}\right)+\operatorname{grad} p=f, \quad x \in \Omega, t \in[0, T], \\
\operatorname{div} v=0, \quad x \in \Omega, t \in[0, T] .
\end{gathered}
$$

This system is obtained from (1.5), (1.6) under the condition $\left(\partial v_{k} / \partial x_{j}\right)\left(\partial / \partial x_{k}\right)\left(\partial v / \partial x_{j}+\right.$ $\left.\operatorname{grad} v_{j}\right) \equiv 0$.

Initial-boundary value problem (1.10), (1.8), (1.9) was studied by Oskolkov in his papers $[11,12]$. However later he remarked in [13] that these papers contain some errors and the obtained results are incorrect. Under the slip boundary condition the system (1.10), has been investigated by Oskolkov in [15]. Ladyzhenskaya notes in her work [7] that the method of introduction of auxiliary viscosity used for study of the initialboundary value problem $(1.10),(1.8),(1.9)$ in the above mentioned papers $[11,12]$ is erroneous and the problem of existence of solutions for the given initial-boundary value problem remains open till now. 
This work is devoted to the study of the initial-boundary value problem (1.5), (1.6), (1.8), and (1.9). Namely, the problem of existence of weak solutions for this problem is investigated.

\section{Principal notations and functional spaces}

Let us introduce the following functional spaces used hereinafter:

$\mathfrak{D}(\Omega)^{n}$ is the space of smooth functions on $\Omega$ with values in $\mathbb{R}^{n}$ and with compact support contained in $\Omega$;

$\mathscr{V}=\left\{v: v \in \mathfrak{D}(\Omega)^{n}, \operatorname{div} v=0\right\}$ is the set of solenoidal smooth functions;

$V$ is the completion of $\mathscr{V}$ in the norm of $W_{2}^{1}(\Omega)^{n}$; we consider the space $V$ with the following norm:

$$
\|v\|_{V}=\left(\int_{\Omega} \nabla v: \nabla v d x\right)^{1 / 2}
$$

Here $\nabla u: \nabla \varphi, u=\left(u_{1}, \ldots, u_{n}\right), \varphi=\left(\varphi_{1}, \ldots, \varphi_{n}\right)$ denotes the component-wise multiplication of matrixes:

$$
\nabla u: \nabla \varphi=\frac{\partial u_{i}}{\partial x_{j}} \cdot \frac{\partial \varphi_{i}}{\partial x_{j}}
$$

In $V$ this norm is equivalent to the norm induced from the space $W_{2}^{1}(\Omega)^{n}$.

$X$ is the completion of $\mathscr{V}$ in the norm of $W_{2}^{3}(\Omega)^{n}$; the norm in $X$ is defined by

$$
\|v\|_{X}=\left(\int_{\Omega} \nabla(\Delta v): \nabla(\Delta v) d x\right)^{1 / 2}
$$

In $X$ this norm is equivalent to the norm induced from the space $W_{2}^{3}(\Omega)^{n}$.

By $X^{*}$ we denote the space conjugate to the space $X$. Denote by $\langle h, v\rangle$ the value of a functional $h \in X^{*}$ on a function $v \in X$.

Denote by $Z$ the image of $X$ under the action of the operator $(I-\varkappa \Delta)$, that is, $Z=$ $(I-\varkappa \Delta) X$. Let us note that $Z$ is a subspace of $V$ but does not coincide with it.

Now we can introduce two principal functional spaces used below

$$
E_{1}=\left\{v: v \in L_{\infty}(0, T ; V), v^{\prime} \in L_{2}\left(0, T ; Z^{*}\right)\right\}
$$

with the norm:

$$
\begin{gathered}
\|v\|_{E_{1}}=\|v\|_{L_{\infty}(0, T ; V)}+\left\|v^{\prime}\right\|_{L_{2}\left(0, T ; Z^{*}\right)}, \\
E_{2}=\left\{v: v \in C([0, T], X), v^{\prime} \in L_{2}(0, T ; X)\right\}
\end{gathered}
$$

with the norm:

$$
\|v\|_{E_{2}}=\|v\|_{C([0, T], X)}+\left\|v^{\prime}\right\|_{L_{2}(0, T ; X)} .
$$




\section{Statement of the problem and the main result}

In this section an approximating problem for the investigated initial-boundary value problem (1.5), (1.6), (1.8), and (1.9) is considered. For this problem and for the original problem the concepts of weak solution is introduced. Then the main result is formulated.

3.1. Approximating problem. For study of the initial-boundary value problem (1.5), (1.6), (1.8), and (1.9) we use a modified method of introduction of auxiliary viscosity. The original method of introduction of auxiliary viscosity for the Navier-Stokes system (see, e.g., Lions [8]) assumes introduction of the additional term $(-1)^{m} \varepsilon \Delta^{m} v$ into the system. Here $\varepsilon>0, m \in \mathbf{N}, m \geqslant(n+2) / 4$. In this work we add the term $-\varepsilon \Delta^{3}(\partial v / \partial t)$ to (1.5). The obtained equations are called approximating equations. Increase of the order of the equation requires introduction of additional boundary conditions.

Consider the following initial-boundary value problem with small parameter $\varepsilon>0$ :

$$
\begin{gathered}
\frac{\partial v}{\partial t}-\varepsilon \Delta^{3}\left(\frac{\partial v}{\partial t}\right)-v \Delta v+v_{i} \frac{\partial v}{\partial x_{i}}-\varkappa \frac{\partial \Delta v}{\partial t}-\varkappa \operatorname{Div}\left(v_{k} \frac{\partial \mathscr{E}(v)}{\partial x_{k}}\right)+\operatorname{grad} p=f, \quad x \in \Omega, t \in[0, T] \\
\operatorname{div} v=0, \quad x \in \Omega, t \in[0, T], \\
v(x, 0)=a(x), \quad x \in \Omega, \\
\left.v\right|_{\partial \Omega \times[0, T]}=0, \\
\left.\frac{\partial v}{\partial n}\right|_{\partial \Omega \times[0, T]}=0, \\
\left.\frac{\partial^{2} v}{\partial n^{2}}\right|_{\partial \Omega \times[0, T]}=0 .
\end{gathered}
$$

Here $\varepsilon$ is some fixed number, $\varepsilon>0 ; n$ is the outer normal to $\partial \Omega$.

Suppose that $f \in L_{2}\left(0, T ; V^{*}\right), a \in X$.

Definition 3.1. A function $v \in E_{2}$ is called a weak solution for the initial-boundary value problem (3.1) if for any $\varphi \in X$ and almost all $t \in(0, T)$ the function $v \in E_{2}$ satisfies the equality

$$
\begin{aligned}
\int_{\Omega} \frac{\partial v}{\partial t} \varphi d x-\int_{\Omega} v_{i} v_{j} \frac{\partial \varphi_{j}}{\partial x_{i}} d x+v \int_{\Omega} \nabla v: \nabla \varphi d x+\varepsilon \int_{\Omega} \nabla\left(\Delta\left(\frac{\partial v}{\partial t}\right)\right): \nabla(\Delta \varphi) d x \\
\quad+\varkappa \int_{\Omega} \nabla\left(\frac{\partial v}{\partial t}\right): \nabla \varphi d x-\frac{\varkappa}{2} \int_{\Omega} v_{k} \frac{\partial v_{i}}{\partial x_{j}} \frac{\partial^{2} \varphi_{j}}{\partial x_{i} \partial x_{k}} d x \\
\quad-\frac{\varkappa}{2} \int_{\Omega} v_{k} \frac{\partial v_{j}}{\partial x_{i}} \frac{\partial^{2} \varphi_{j}}{\partial x_{i} \partial x_{k}} d x=\langle f, \varphi\rangle
\end{aligned}
$$

and the initial condition

$$
v(0)=a
$$


3.2. Definition of weak solution for initial-boundary value problem (1.5), (1.6), (1.8), and (1.9). Suppose that $f \in L_{2}\left(0, T ; V^{*}\right), a_{*} \in V$.

Definition 3.2. A function $v \in E_{1}$ is a weak solution for the initial-boundary value problem (1.5), (1.6), (1.8), and (1.9) if for any $\varphi \in X$ and almost all $t \in(0, T)$ the function $v$ satisfies the equality

$$
\begin{aligned}
& \left\langle(I-\varkappa \Delta) \frac{\partial v}{\partial t}, \varphi\right\rangle-\int_{\Omega} v_{i} v_{j} \frac{\partial \varphi_{j}}{\partial x_{i}} d x+v \int_{\Omega} \nabla v: \nabla \varphi d x \\
& -\frac{\varkappa}{2} \int_{\Omega} v_{k} \frac{\partial v_{i}}{\partial x_{j}} \frac{\partial^{2} \varphi_{j}}{\partial x_{i} \partial x_{k}} d x-\frac{\varkappa}{2} \int_{\Omega} v_{k} \frac{\partial v_{j}}{\partial x_{i}} \frac{\partial^{2} \varphi_{j}}{\partial x_{i} \partial x_{k}} d x=\langle f, \varphi\rangle
\end{aligned}
$$

and the initial condition

$$
v(0)=a_{*}
$$

3.3. The main result. Our main result is the following theorem.

Theorem 3.3. For any $f \in L_{2}\left(0, T ; V^{*}\right), a \in V$ the initial-boundary value problem (1.5), (1.6), (1.8), and (1.9) has at least one weak solution $v_{*} \in E_{1}$.

In order to prove this theorem we use the modified method of introduction of auxiliary viscosity. At the first stage we introduce into (1.5) the term $-\varepsilon \Delta^{3}(\partial v / \partial t)$. The resolvability of the obtained approximating problem is established by topological methods on the basis of a priori estimates of weak solutions. Then it is shown that in a sequence of weak solutions of the approximating problem it is possible to select a subsequence converging to a weak solution of the initial-boundary value problem (1.5), (1.6), (1.8), and (1.9) as the parameter of approximation tends to zero.

\section{Operator treatment for the approximating problem (3.1)}

Let us introduce operators in functional spaces using the following equalities:

$$
\begin{gathered}
A: V \rightarrow V, \quad\langle A v, \varphi\rangle=\int_{\Omega} \nabla v: \nabla \varphi d x, \quad v, \varphi \in V, \\
N: X \rightarrow X^{*}, \quad\langle N v, \varphi\rangle=\int_{\Omega} \nabla(\Delta v): \nabla(\Delta \varphi) d x, \quad v, \varphi \in X, \\
B_{1}: L_{4}(\Omega)^{n} \longrightarrow V^{*}, \quad\left\langle B_{1}(v), \varphi\right\rangle=\int_{\Omega} v_{i} v_{j} \frac{\partial \varphi_{j}}{\partial x_{i}} d x, \quad v \in L_{4}(\Omega)^{n}, \varphi \in V, \\
B_{2}: V \longrightarrow X^{*}, \quad\left\langle B_{2}(v), \varphi\right\rangle=\int_{\Omega} v_{k} \frac{\partial v_{i}}{\partial x_{j}} \frac{\partial^{2} \varphi_{j}}{\partial x_{i} \partial x_{k}} d x, \quad v \in V, \varphi \in X, \\
B_{3}: V \longrightarrow X^{*}, \quad\left\langle B_{3}(v), \varphi\right\rangle=\int_{\Omega} v_{k} \frac{\partial v_{j}}{\partial x_{i}} \frac{\partial^{2} \varphi_{j}}{\partial x_{i} \partial x_{k}} d x, \quad v \in V, \varphi \in X, \\
J: X \longrightarrow X^{*}, \quad\langle J v, \varphi\rangle=\int_{\Omega} v \varphi d x, \quad v, \varphi \in X .
\end{gathered}
$$


Since in (3.2) the function $\varphi \in X$ is arbitrary, this equation is equivalent to the following operator equation:

$$
J v^{\prime}-B_{1}(v)+v A v+\varepsilon N v^{\prime}+\varkappa A v^{\prime}-\frac{\varkappa}{2} B_{2}(v)-\frac{\varkappa}{2} B_{3}(v)=f .
$$

Hence the problem of existence of a weak solution for the problem (3.1) is equivalent to the problem of existence of a solution $v \in E_{2}$ for operator equation (4.2) satisfying the initial condition (3.3).

4.1. Properties of operators $A$ and $N$. In this subsection the properties of operators $A$ and $N$ from the operator equation (4.2) are investigated.

Lemma 4.1. The operator $A$ has the following properties.

(i) The operator $A: V \rightarrow V^{*}$ is continuous and the following inequality holds:

$$
\|A u\|_{V} \leqslant\|u\|_{V^{*}}, \quad u \in V
$$

(ii) For any $u \in L_{2}(0, T ; V)$ one has $A u \in L_{2}\left(0, T ; V^{*}\right)$ and the operator $A: L_{2}(0, T ; V) \rightarrow$ $L_{2}\left(0, T ; V^{*}\right)$ is continuous.

(iii) For any $u \in E_{2}$ one has $A u \in L_{2}\left(0, T ; X^{*}\right)$ and the operator $A: E_{2} \rightarrow L_{2}\left(0, T ; X^{*}\right)$ is compact and the following estimate holds:

$$
\|A u\|_{L_{2}\left(0, T ; X^{*}\right)} \leqslant C_{0}\|u\|_{C([0, T], V)} .
$$

Proof. (i) It suffices to show boundedness of the linear operator $A$. By definition we have

$$
|\langle A u, \varphi\rangle|=\left|\int_{\Omega} \nabla u: \nabla \varphi d x\right| \leqslant\|u\|_{V}\|\varphi\|_{V}
$$

The last inequality implies (4.3). Thus the operator $A: V \rightarrow V^{*}$ is continuous.

(ii) Let $u \in L_{2}(0, T ; V)$. For almost all $t \in(0, T)$ by virtue of (4.3) we have

$$
\|A u(t)\|_{V^{*}}^{2} \leqslant\|u(t)\|_{V^{*}}^{2}
$$

Therefore $A u \in L_{2}\left(0, T ; V^{*}\right)$.

Integrating the obtained estimate with respect to $t$ from 0 to $T$, we obtain

$$
\|A u\|_{L_{2}\left(0, T ; V^{*}\right)}^{2} \leqslant\|u\|_{L_{2}(0, T ; V)}^{2} .
$$

Since the operator $A$ is linear and bounded, it is continuous as an operator from $L_{2}(0, T$; $V)$ to $L_{2}\left(0, T ; V^{*}\right)$.

(iii) In order to prove that the operator $A: E_{2} \rightarrow L_{2}\left(0, T ; X^{*}\right)$ is compact we use [17, Theorem 2.1, page 184]. We remind here its statement. 
Theorem 4.2. Let $X_{0}, F, X_{1}$ be three Banach spaces satisfying the following condition:

$$
X_{0} \subset F \subset X_{1}
$$

Where the embeddings are continuous, $X_{0}, X_{1}$ are reflexive, the embedding $X_{0} \rightarrow F$ is compact. Let $T>0$ be fixed finite number and $\alpha_{0}, \alpha_{1}$ be two finite numbers such that $\alpha_{i}>1$, $i=0,1$.

Suppose that $Y=\left\{v: v \in L_{\alpha_{0}}\left(0, T ; X_{0}\right) ; v^{\prime} \in L_{\alpha_{1}}\left(0, T ; X_{1}\right)\right\}$ is the space with the norm $\|v\|_{Y}=\|v\|_{L_{\alpha_{0}}\left(0, T ; X_{0}\right)}+\left\|v^{\prime}\right\|_{L_{\alpha_{1}}\left(0, T ; X_{1}\right)}$. Then the embedding of $Y$ into $L_{\alpha_{0}}(0, T ; F)$ is compact.

In our case

$$
\begin{gathered}
X_{0}=X, \quad F=V, \quad X_{1}=L_{2}(\Omega)^{n}, \quad \alpha_{0}=\alpha_{1}=2, \\
Y=\left\{v: v \in L_{2}(0, T ; X) ; v^{\prime} \in L_{2}\left(0, T ; L_{2}(\Omega)^{n}\right)\right\} .
\end{gathered}
$$

Since the embedding $X=V \cap W_{2}^{3}(\Omega)^{n} \subset V$ is compact, all conditions of this theorem hold and consequently the embedding of $Y$ into $L_{2}(0, T ; V)$ is compact.

Note that $E_{2} \subset Y$ and this embedding is continuous. Indeed, it follows from the embeddings $C([0, T], X) \subset L_{2}(0, T ; X)$ and $L_{2}(0, T ; X) \subset L_{2}\left(0, T ; L_{2}(\Omega)^{n}\right)$ which are continuous. From (ii) we have that the operator $A: L_{2}(0, T ; V) \rightarrow L_{2}\left(0, T ; V^{*}\right)$ is continuous.

Thus we have the following composition

$$
E_{2} \subset Y \subset L_{2}(0, T ; V) \stackrel{A}{\longrightarrow} L_{2}\left(0, T ; V^{*}\right) \subset L_{2}\left(0, T ; X^{*}\right) .
$$

Here the first embedding is continuous, the second embedding is compact, and the map $A$ and the last embedding are continuous. This yields that the map $A: E_{2} \rightarrow L_{2}\left(0, T ; X^{*}\right)$ is completely continuous.

The required estimate follows from (4.7) and continuity of embeddings $L_{2}\left(0, T ; V^{*}\right) \subset$ $L_{2}\left(0, T ; X^{*}\right)$ and $C([0, T], V) \subset L_{2}(0, T ; V)$.

LEMma 4.3. The operator $J+\varepsilon N+\varkappa A$ has the following properties.

(i) The operator $(J+\varepsilon N+\varkappa A): X \rightarrow X^{*}$ is continuous, invertible and the following inequalities hold

$$
\varepsilon\|u\|_{X} \leqslant\|(J+\varepsilon N+\varkappa A) u\|_{X^{*}} \leqslant\left(C_{1}+\varepsilon+\varkappa C_{2}\right)\|u\|_{X}
$$

Here $C_{1}, C_{2}$ are some constants, which depend from $n$ and domain $\Omega$ and do not depend on $u$.

(ii) For any $u \in L_{2}(0, T ; X)$ one has $(J+\varepsilon N+\varkappa A) u \in L_{2}\left(0, T ; X^{*}\right)$ and the operator $(J+\varepsilon N+\varkappa A): L_{2}(0, T ; X) \rightarrow L_{2}\left(0, T ; X^{*}\right)$ is continuous, invertible and the following estimate holds

$$
\varepsilon\|u\|_{L_{2}(0, T ; X)} \leqslant\|(J+\varepsilon N+\varkappa A) u\|_{L_{2}\left(0, T ; X^{*}\right)} .
$$


Proof. (i) By linearity of the operator $J+\varepsilon N+\varkappa A$ for the proof of its continuity it suffices to show its boundedness. By definition we have

$$
\begin{aligned}
|\langle(J+\varepsilon N+\varkappa A) u, \varphi\rangle| & =\left|\int_{\Omega} u \varphi d x+\varepsilon \int_{\Omega} \nabla(\Delta u): \nabla(\Delta \varphi) d x+\varkappa \int_{\Omega} \nabla u: \nabla \varphi d x\right| \\
& \leqslant\|u\|_{L_{2}(\Omega)^{n}}\|\varphi\|_{L_{2}(\Omega)^{n}}+\varepsilon\|u\|_{X}\|\varphi\|_{X}+\varkappa\|u\|_{V}\|\varphi\|_{V} \\
& \leqslant C_{1}\|u\|_{X}\|\varphi\|_{X}+\varepsilon\|u\|_{X}\|\varphi\|_{X}+\varkappa C_{2}\|u\|_{X}\|\varphi\|_{X} \\
& =\left(C_{1}+\varepsilon+\varkappa C_{2}\right)\|u\|_{X}\|\varphi\|_{X} .
\end{aligned}
$$

We have used two following inequalities:

$$
\|u\|_{L_{2}(\Omega)^{n}}^{2} \leqslant C_{1}\|u\|_{X}^{2}, \quad\|u\|_{V}^{2} \leqslant C_{2}\|u\|_{X}^{2} .
$$

Here the constants $C_{1}, C_{2}$ depend on $n$ and domain $\Omega$ and do not depend on $u$.

This implies the right part of estimation (4.11). Thus the operator $(J+\varepsilon N+\varkappa A): X \rightarrow$ $X^{*}$ is bounded and hence it is continuous.

In order to prove that the operator $(I+\varepsilon N+\varkappa A): X \rightarrow X^{*}$ is invertible we use [17, Theorem 2.2, page 17]. We remind here its statement.

Theorem 4.4. Let $W$ be a separable real Hilbert space (norm $\|\cdot\|_{W}$ ) and let $a(u, v)$ be a linear continuous form on $W \times W$, which is coercive, that is, there exists $\alpha>0$, such that

$$
a(u, u) \geqslant \alpha\|u\|_{W}^{2} \quad \forall u \in W .
$$

Then for each $l$ from $W^{*}$, the dual space of $W$, there exists one and only one $u \in W$ such that

$$
a(u, v)=\langle l, v\rangle \quad \forall v \in W .
$$

To apply this theorem it suffices to show that the following continuous bilinear form is coercive.

$$
a(u, v)=\langle(J+\varepsilon N+\varkappa A) u, v\rangle=\int_{\Omega} u v d x+\varepsilon \int_{\Omega} \nabla(\Delta u): \nabla(\Delta v) d x+\varkappa \int_{\Omega} \nabla u: \nabla v d x .
$$

In fact, for any $u \in X$ we have

$$
\begin{aligned}
a(u, u) & =\langle(J+\varepsilon N+\varkappa A) u, u\rangle \\
& =\int_{\Omega} u^{2} d x+\varepsilon \int_{\Omega} \nabla(\Delta u): \nabla(\Delta u) d x+\varkappa \int_{\Omega} \nabla u: \nabla u d x \\
& =\|u\|_{L_{2}(\Omega)^{n}}^{2}+\varepsilon\|u\|_{X}^{2}+\varkappa\|u\|_{V}^{2} \geq \varepsilon\|u\|_{X}^{2}, \quad \varepsilon>0 .
\end{aligned}
$$

Thus we have proved that $(J+\varepsilon N+\varkappa A): X \rightarrow X^{*}$ is an isomorphism and the left part of estimate (4.11) is valid. 
Furthermore by virtue of $\left[4\right.$, Remark 2.3] the inverse operator $(J+\varepsilon N+\varkappa A)^{-1}: X^{*} \rightarrow$ $X$ is Lipschitz-continuous (in the nomenclature of [4]). That is for any $f, g \in X^{*}$ :

$$
\left\|(J+\varepsilon N+\varkappa A)^{-1} f-(J+\varepsilon N+\varkappa A)^{-1} g\right\|_{X^{*}} \leqslant C_{3}\|f-g\|_{X}, \quad C_{3}=\frac{1}{\varepsilon} .
$$

(ii) Let $u \in L_{2}(0, T ; X)$. Quadrating right part of estimate (4.11) at almost all $t$ and integrating with respect to $t$ from 0 to $T$, we get

$$
\int_{0}^{T}\|(J+\varepsilon N+\varkappa A) u(t)\|_{X^{*}}^{2} d t \leqslant\left(C_{1}+\varepsilon+\varkappa C_{2}\right)^{2}\|u\|_{L_{2}(0, T ; X)}^{2}
$$

The right-hand side of this inequality is finite and therefore the left-hand side is also finite. From here it follows that $(J+\varepsilon N+\varkappa A) u \in L_{2}\left(0, T ; X^{*}\right)$ and

$$
\|(J+\varepsilon N+\varkappa A) u\|_{L_{2}\left(0, T ; X^{*}\right)} \leqslant\left(C_{1}+\varepsilon+\varkappa C_{2}\right)\|u\|_{L_{2}(0, T ; X)}
$$

The operator $(J+\varepsilon N+\varkappa A): L_{2}(0, T ; X) \rightarrow L_{2}\left(0, T ; X^{*}\right)$ is linear and bounded and therefore it is continuous.

Now, let us show that it is invertible. First we will prove that the range of the operator $(J+\varepsilon N+\varkappa A)$ coincides with $L_{2}\left(0, T ; X^{*}\right)$. For this purpose it is necessary to show that for any $w \in L_{2}\left(0, T ; X^{*}\right)$ the equation $(J+\varepsilon N+\varkappa A) u=w$ has a solution $u \in L_{2}(0, T ; X)$. By (i) the operator $(J+\varepsilon N+\varkappa A): X \rightarrow X^{*}$ is invertible. This implies that al almost all $t \in(0, T)$ the equation $(J+\varepsilon N+\varkappa A) u=w$ has a solution $u(t)=(J+\varepsilon N+\varkappa A)^{-1} w(t)$. It is necessary to show that the function $u \in L_{2}(0, T ; X)$. Using the left part of the estimate (4.11) we get

$$
\varepsilon\|u(t)\|_{X} \leqslant\|(J+\varepsilon N+\varkappa A) u(t)\|_{X^{*}}=\|w(t)\|_{X^{*}} .
$$

Since $w \in L_{2}\left(0, T ; X^{*}\right)$ we have from this inequality that $u \in L_{2}(0, T ; X)$. Quadrating this inequality and integrating it along $(0, T)$, we obtain the required inequality (4.12).

Therefore $\operatorname{ker}(J+\varepsilon N+\varkappa A)=\{0\}$. Thus the operator $(J+\varepsilon N+\varkappa A): L_{2}(0, T ; X) \rightarrow$ $L_{2}\left(0, T ; X^{*}\right)$ is invertible.

4.2. Properties of operators $B_{1}, B_{2}, B_{3}$. In this section we consider the properties of operators $B_{1}, B_{2}$ and $B_{3}$ from (4.2).

Lemma 4.5. The map $B_{1}$ has the following properties.

(i) The map $B_{1}: L_{4}(\Omega)^{n} \rightarrow V^{*}$ is continuous and the following inequality holds

$$
\left\|B_{1}(v)\right\|_{V^{*}} \leqslant C_{4}\|v\|_{L_{4}(\Omega)^{n}}^{2} .
$$

(ii) For any $v \in L_{4}\left(0, T ; L_{4}(\Omega)^{n}\right)$ one has $B_{1}(v) \in L_{2}\left(0, T ; V^{*}\right)$ and the map $B_{1}: L_{4}(0, T$; $\left.L_{4}(\Omega)^{n}\right) \rightarrow L_{2}\left(0, T ; V^{*}\right)$ is continuous. 
(iii) For any $v \in E_{2}$ one has $B_{1}(v) \in L_{2}\left(0, T ; X^{*}\right)$, the map $B_{1}: E_{2} \rightarrow L_{2}\left(0, T ; X^{*}\right)$ is compact and the following estimate holds:

$$
\left\|B_{1}(v)\right\|_{L_{2}\left(0, T ; X^{*}\right)} \leqslant C_{5}\|v\|_{C([0, T], V)}^{2} .
$$

Proof. (i) Let $v \in L_{4}(\Omega)^{n}, \varphi \in V$. Then we obtain

$$
\begin{aligned}
\left|\left\langle B_{1}(v), \varphi\right\rangle\right| & =\left|\int_{\Omega} v_{i} v_{j} \frac{\partial \varphi_{j}}{\partial x_{i}} d x\right| \leqslant \sum_{i, j=1}^{n}\left\|v_{i}\right\|_{L_{4}(\Omega)}\left\|v_{j}\right\|_{L_{4}(\Omega)}\left\|\frac{\partial \varphi_{j}}{\partial x_{i}}\right\|_{L_{2}(\Omega)} \\
& \leqslant \sum_{i, j=1}^{n}\|v\|_{L_{4}(\Omega)^{n}}\|v\|_{L_{4}(\Omega)^{n}}\|\varphi\|_{V} \leqslant n^{2}\|v\|_{L_{4}(\Omega)^{n}}^{2}\|\varphi\|_{V} \\
& \leqslant C_{4}\|v\|_{L_{4}(\Omega)^{n}}^{2}\|\varphi\|_{V} .
\end{aligned}
$$

Whence the required estimation (4.23) follows.

Let us prove that the map $B_{1}: L_{4}(\Omega)^{n} \rightarrow V^{*}$ is continuous. For any $v^{m}, v^{0} \in L_{4}(\Omega)^{n}$ we obtain

$$
\begin{aligned}
\left|\left\langle B_{1}\left(v^{m}\right), \varphi\right\rangle-\left\langle B_{1}\left(v^{0}\right), \varphi\right\rangle\right| & =\left|\int_{\Omega} v_{i}^{m} v_{j}^{m} \frac{\partial \varphi_{j}}{\partial x_{i}} d x-\int_{\Omega} v_{i}^{0} v_{j}^{0} \frac{\partial \varphi_{j}}{\partial x_{i}} d x\right| \\
& \leqslant \sum_{i, j=1}^{n}\left\|v_{i}^{m} v_{j}^{m}-v_{i}^{0} v_{j}^{0}\right\|_{L_{2}(\Omega)}\left\|\frac{\partial \varphi_{j}}{\partial x_{i}}\right\|_{L_{2}(\Omega)} \\
& \leqslant\|\varphi\|_{V^{*}} \sum_{i, j=1}^{n}\left\|v_{i}^{m} v_{j}^{m}-v_{i}^{0} v_{j}^{0}\right\|_{L_{2}(\Omega)} .
\end{aligned}
$$

We obviously have

$$
\begin{aligned}
\sum_{i, j=1}^{n}\left\|v_{i}^{m} v_{j}^{m}-v_{i}^{0} v_{j}^{0}\right\|_{L_{2}(\Omega)} & =\sum_{i, j=1}^{n}\left\|v_{i}^{m} v_{j}^{m}-v_{i}^{m} v_{j}^{0}+v_{i}^{m} v_{j}^{0}-v_{i}^{0} v_{j}^{0}\right\|_{L_{2}(\Omega)} \\
& \leqslant \sum_{i, j=1}^{n}\left\|v_{i}^{m}\left(v_{j}^{m}-v_{j}^{0}\right)\right\|_{L_{2}(\Omega)}+\sum_{i, j=1}^{n}\left\|v_{j}^{0}\left(v_{i}^{m}-v_{i}^{0}\right)\right\|_{L_{2}(\Omega)} \\
& \leqslant \sum_{i, j=1}^{n}\left\|v_{i}^{m}\right\|_{L_{4}(\Omega)}\left\|v_{j}^{m}-v_{j}^{0}\right\|_{L_{4}(\Omega)}+\sum_{i, j=1}^{n}\left\|v_{j}^{0}\right\|_{L_{4}(\Omega)}\left\|v_{i}^{m}-v_{i}^{0}\right\|_{L_{4}(\Omega)} \\
& \leqslant C_{6}\left\|v^{m}\right\|_{L_{4}(\Omega)^{n}}\left\|v^{m}-v^{0}\right\|_{L_{4}(\Omega)^{n}}+C_{6}\left\|v^{0}\right\|_{L_{4}(\Omega)^{n}}\left\|v^{m}-v^{0}\right\|_{L_{4}(\Omega)^{n}} \\
& =C_{6}\left(\left\|v^{m}\right\|_{L_{4}(\Omega)^{n}}+\left\|v^{0}\right\|_{L_{4}(\Omega)^{n}}\right)\left\|v^{m}-v^{0}\right\|_{L_{4}(\Omega)^{n}} .
\end{aligned}
$$

Therefore

$$
\left\|B_{1}\left(v^{m}\right)-B_{1}\left(v^{0}\right)\right\|_{V^{*}} \leq C_{6}\left(\left\|v^{m}\right\|_{L_{4}(\Omega)^{n}}+\left\|v^{0}\right\|_{L_{4}(\Omega)^{n}}\right)\left\|v^{m}-v^{0}\right\|_{L_{4}(\Omega)^{n}} .
$$

Let a sequence $\left\{v^{m}\right\} \subset L_{4}(\Omega)^{n}$ converge to some limit function $v^{0} \in L_{4}(\Omega)^{n}$. Then the continuity of the map $B_{1}: L_{4}(\Omega)^{n} \rightarrow V^{*}$ follows from inequality (4.28). 
(ii) Let $v \in L_{4}\left(0, T ; L_{4}(\Omega)^{n}\right)$. Quadrating estimate (4.23) and integrating with respect to $t$ from 0 to $T$, we get

$$
\int_{0}^{T}\left\|B_{1}(v)(t)\right\|_{V^{*}}^{2} d t \leq C_{4}^{2} \int_{0}^{T}\|v(t)\|_{L_{4}(\Omega)^{n}}^{4} d t=C_{4}^{2}\|v\|_{L_{4}\left(0, T ; L_{4}(\Omega)^{n}\right)}^{4} .
$$

Since $v \in L_{4}\left(0, T ; L_{4}(\Omega)^{n}\right)$, one has $\left\|B_{1}(v)(t)\right\|_{V^{*}} \in L_{2}(0, T)$ and hence $B_{1}(v) \in L_{2}(0, T$; $\left.V^{*}\right)$.

Let us prove that the map $B_{1}: L_{4}\left(0, T ; L_{4}(\Omega)^{n}\right) \rightarrow L_{2}\left(0, T ; V^{*}\right)$ is continuous.

Let a sequence $\left\{v^{m}\right\} \subset L_{4}\left(0, T ; L_{4}(\Omega)^{n}\right)$ converge to some limit $v^{0} \in L_{4}\left(0, T ; L_{4}(\Omega)^{n}\right)$. Quadrating inequality (4.28) and integrating with respect to $t$ from 0 to $T$, we get

$$
\begin{aligned}
\int_{0}^{T} \| & B_{1}\left(v^{m}(t)\right)-B_{1}\left(v^{0}(t)\right) \|_{V^{*}}^{2} d t \\
\leqslant & C_{6}^{2} \int_{0}^{T}\left(\left\|v^{m}(t)\right\|_{L_{4}(\Omega)^{n}}+\left\|v^{0}(t)\right\|_{L_{4}(\Omega)^{n}}\right)^{2}\left\|v^{m}(t)-v^{0}(t)\right\|_{L_{4}(\Omega)^{n}}^{2} d t \\
\leqslant & C_{6}^{2}\left(\int_{0}^{T}\left(\left\|v^{m}(t)\right\|_{L_{4}(\Omega)^{n}}+\left\|v^{0}(t)\right\|_{L_{4}(\Omega)^{n}}\right)^{4} d t\right)^{1 / 2}\left(\int_{0}^{T}\left\|v^{m}(t)-v^{0}(t)\right\|_{L_{4}(\Omega)^{n}}^{4} d t\right)^{1 / 2} \\
= & C_{6}^{2}\left(\int_{0}^{T} \sum_{i=0}^{4} C_{4}^{i}\left\|v^{m}(t)\right\|_{L_{4}(\Omega)^{n}}^{i}\left\|v^{0}(t)\right\|_{L_{4}(\Omega)^{n}}^{4-i} d t\right)^{1 / 2}\left(\int_{0}^{T}\left\|v^{m}(t)-v^{0}(t)\right\|_{L_{4}(\Omega)^{n}}^{4} d t\right)^{1 / 2} \\
\leqslant & C_{6}^{2}\left(\sum_{i=0}^{4} C_{4}^{i}\left(\int_{0}^{T}\left\|v^{m}(t)\right\|_{L_{4}(\Omega)^{n}}^{4} d t\right)^{i / 4}\left(\int_{0}^{T}\left\|v^{0}(t)\right\|_{L_{4}(\Omega)^{n}}^{4} d t\right)^{(4-i) / 4}\right)^{1 / 2} \\
& \times\left(\int_{0}^{T}\left\|v^{m}(t)-v^{0}(t)\right\|_{L_{4}(\Omega)^{n}}^{4} d t\right)^{1 / 2} \cdot
\end{aligned}
$$

Thus we obtain

$$
\begin{aligned}
& \left\|B_{1}\left(v^{m}\right)-B_{1}\left(v^{0}\right)\right\|_{L_{2}\left(0, T ; V^{*}\right)}^{2} \\
& \quad \leqslant C_{6}^{2}\left(\sum_{i=0}^{4} C_{4}^{i}\left\|v^{m}\right\|_{L_{4}\left(0, T ; L_{4}(\Omega)^{n}\right)}^{i / 4}\left\|v^{0}\right\|_{L_{4}\left(0, T ; L_{4}(\Omega)^{n}\right)}^{(4-i) / 4}\right)^{2}\left\|v^{m}-v^{0}\right\|_{L_{4}\left(0, T ; L_{4}(\Omega)^{n}\right)^{n}}^{2} .
\end{aligned}
$$

Here $C_{4}^{i}=4 ! / i !(4-i)$ !. Since the right-hand side of (4.31) tends to zero as $m \rightarrow+\infty$, the left-hand side tends to zero as $m \rightarrow+\infty$. This completes the proof of (ii).

(iii) Let us use [17, Theorem 2.1, page 184]. We quoted its statement in Lemma 4.1. In our case

$$
\begin{gathered}
X_{0}=X, \quad F=L_{4}(\Omega)^{n}, \quad X_{1}=L_{2}(\Omega)^{n}, \quad \alpha_{0}=4, \quad \alpha_{1}=2, \\
Y=\left\{v: v \in L_{4}(0, T ; X) ; v^{\prime} \in L_{2}\left(0, T ; L_{2}(\Omega)^{n}\right)\right\} .
\end{gathered}
$$

By Sobolev's embedding theorem we have compact embedding $X \subset L_{4}(\Omega)^{n}$. All conditions of [17, Theorem 2.1] hold and we get that the embedding of $Y$ into $L_{4}(0, T$; $\left.L_{4}(\Omega)^{n}\right)$ is compact. 
Since $C([0, T], X) \subset L_{4}\left(0, T ; L_{4}(\Omega)^{n}\right)$ and $C([0, T], X) \subset L_{2}\left(0, T ; L_{2}(\Omega)^{n}\right)$ and these embeddings are continuous, $E_{2} \subset Y$ and this embedding is continuous. By (ii) the map $B_{1}: L_{4}\left(0, T ; L_{4}(\Omega)^{n}\right) \rightarrow L_{2}\left(0, T ; V^{*}\right)$ is continuous.

Finally, we obtain

$$
E_{2} \subset Y \subset L_{4}\left(0, T ; L_{4}(\Omega)^{n}\right) \stackrel{B_{1}}{\longrightarrow} L_{2}\left(0, T ; V^{*}\right) \subset L_{2}\left(0, T ; X^{*}\right) .
$$

Here the first embedding is continuous, the second embedding is compact and the map $B_{1}$ and the last embedding are continuous. Thus for any $v \in E_{2}$ we have that $B_{1}(v) \in$ $L_{2}\left(0, T ; X^{*}\right)$ and the map $B_{1}: X \rightarrow L_{2}\left(0, T ; X^{*}\right)$ is compact.

Combining (4.29) and the continuous embeddings $C([0, T], V) \subset L_{4}\left(0, T ; L_{4}(\Omega)^{n}\right)$ and $L_{2}\left(0, T ; V^{*}\right) \subset L_{2}\left(0, T ; X^{*}\right)$ we have the required estimate (4.24).

Lemma 4.6. The operators $B_{2}$ and $B_{3}$ have the following properties.

(i) For $i=2,3$ the operator $B_{i}: V \rightarrow X^{*}$ is continuous and the following inequality holds:

$$
\left\|B_{i}(v)\right\|_{X^{*}} \leqslant C_{7}\|v\|_{V}^{2}
$$

(ii) Let $i=2,3$ and $v \in L_{4}(0, T ; V)$, then $B_{i}(v) \in L_{2}\left(0, T ; X^{*}\right)$ and the map $B_{i}: L_{4}(0$, $T ; V) \rightarrow L_{2}\left(0, T ; X^{*}\right)$ is continuous.

(iii) Let $i=2,3$ and $v \in E_{2}$, then $B_{i}(v) \in L_{2}\left(0, T ; X^{*}\right)$, the map $B_{i}: E_{2} \rightarrow L_{2}\left(0, T ; X^{*}\right)$ is compact and the following estimate holds:

$$
\left\|B_{i}(v)\right\|_{L_{2}\left(0, T ; X^{*}\right)} \leqslant C_{7} \sqrt{T}\|v\|_{C([0, T], V)}^{2} .
$$

Proof. Let us prove this lemma for the operator $B_{2}$. The proof in case of the operator $B_{3}$ is completely similar.

(i) For any $v \in V, \varphi \in X$ by definition of the operator $B_{2}$ we have

$$
\begin{aligned}
\left|\left\langle B_{2}(v), \varphi\right\rangle\right| & =\left|\int_{\Omega} v_{k} \frac{\partial v_{i}}{\partial x_{j}} \frac{\partial^{2} \varphi_{j}}{\partial x_{i} \partial x_{k}} d x\right| \leqslant \sum_{i, j, k=1}^{n}\left\|v_{k}\right\|_{L_{4}(\Omega)}\left\|\frac{\partial v_{i}}{\partial x_{j}}\right\|_{L_{2}(\Omega)}\left\|\frac{\partial^{2} \varphi_{j}}{\partial x_{i} \partial x_{k}}\right\| \|_{L_{4}(\Omega)} \\
& \leq \sum_{i, j, k=1}^{n}\|v\|_{L_{4}(\Omega)^{n}}\|v\|_{V}\|\varphi\|_{X} \leqslant C_{7}\|v\|_{V}^{2}\|\varphi\|_{X} .
\end{aligned}
$$

This implies the required estimate (4.34).

Let us prove continuity of the map $B_{2}: V \rightarrow X^{*}$. For any $v^{m}, v^{0} \in V$ we get

$$
\begin{aligned}
\left|\left\langle B_{2}\left(v^{m}\right), \varphi\right\rangle-\left\langle B_{2}\left(v^{0}\right), \varphi\right\rangle\right| & =\left|\int_{\Omega} v_{k}^{m} \frac{\partial v_{i}^{m}}{\partial x_{j}} \frac{\partial^{2} \varphi_{j}}{\partial x_{i} \partial x_{k}} d x-\int_{\Omega} v_{k}^{0} \frac{\partial v_{i}^{0}}{\partial x_{j}} \frac{\partial^{2} \varphi_{j}}{\partial x_{i} \partial x_{k}} d x\right| \\
& \leq \sum_{i, j, k=1}^{n}\left\|v_{k}^{m} \frac{\partial v_{i}^{m}}{\partial x_{j}}-v_{k}^{0} \frac{\partial v_{i}^{0}}{\partial x_{j}}\right\|_{L_{4 / 3}(\Omega)}\left\|\frac{\partial^{2} \varphi_{j}}{\partial x_{i} \partial x_{k}}\right\| \|_{L_{4}(\Omega)} \\
& \leq \sum_{i, j, k=1}^{n}\left\|v_{k}^{m} \frac{\partial v_{i}^{m}}{\partial x_{j}}-v_{k}^{0} \frac{\partial v_{i}^{0}}{\partial x_{j}}\right\|_{L_{4 / 3}(\Omega)}\|\varphi\|_{X} .
\end{aligned}
$$


Hence

$$
\left\|B_{2}\left(v^{m}\right)-B_{2}\left(v^{0}\right)\right\|_{X^{*}} \leq \sum_{i, j, k=1}^{n}\left\|v_{k}^{m} \frac{\partial v_{i}^{m}}{\partial x_{j}}-v_{k}^{0} \frac{\partial v_{i}^{0}}{\partial x_{j}}\right\|_{L_{4 / 3}(\Omega)} .
$$

Let us transform a right-hand side of this inequality as follows:

$$
\begin{aligned}
\left\|v_{k}^{m} \frac{\partial v_{i}^{m}}{\partial x_{j}}-v_{k}^{0} \frac{\partial v_{i}^{0}}{\partial x_{j}}\right\|_{L_{4 / 3}(\Omega)}= & \sum_{i, j, k=1}^{n}\left\|v_{k}^{m} \frac{\partial v_{i}^{m}}{\partial x_{j}}-v_{k}^{m} \frac{\partial v_{i}^{0}}{\partial x_{j}}+v_{k}^{m} \frac{\partial v_{i}^{0}}{\partial x_{j}}-v_{k}^{0} \frac{\partial v_{i}^{0}}{\partial x_{j}}\right\|_{L_{4 / 3}(\Omega)} \\
\leq & \sum_{i, j, k=1}^{n}\left\|v_{k}^{m} \frac{\partial v_{i}^{m}}{\partial x_{j}}-v_{k}^{m} \frac{\partial v_{i}^{0}}{\partial x_{j}}\right\|_{L_{4 / 3}(\Omega)}+\sum_{i, j, k=1}^{n}\left\|v_{k}^{m} \frac{\partial v_{i}^{0}}{\partial x_{j}}-v_{k}^{0} \frac{\partial v_{i}^{0}}{\partial x_{j}}\right\|_{L_{4 / 3}(\Omega)} \\
\leq & \sum_{i, j, k=1}^{n}\left\|v_{k}^{m}\right\|_{L_{4}(\Omega)}\left\|\frac{\partial v_{i}^{m}}{\partial x_{j}}-\frac{\partial v_{i}^{0}}{\partial x_{j}}\right\|_{L_{2}(\Omega)} \\
& +\sum_{i, j, k=1}^{n}\left\|\frac{\partial v_{i}^{0}}{\partial x_{j}}\right\|\left\|_{L_{2}(\Omega)}\right\| v_{k}^{m}-v_{k}^{0} \|_{L_{4}(\Omega)} \\
\leq & n^{3}\left\|v^{m}\right\|_{L_{4}(\Omega)^{n}}\left\|v^{m}-v^{0}\right\|_{V}+n^{3}\left\|v^{0}\right\|_{V}\left\|v^{m}-v^{0}\right\|_{L_{4}(\Omega)^{n}} \\
\leq & C_{8}\left(\left\|v^{m}\right\|_{V}+\left\|v^{0}\right\|_{V}\right)\left\|v^{m}-v^{0}\right\|_{V} .
\end{aligned}
$$

Therefore we have

$$
\left\|B_{2}\left(v^{m}\right)-B_{2}\left(v^{0}\right)\right\|_{X^{*}} \leqslant C_{8}\left(\left\|v^{m}\right\|_{V}+\left\|v^{0}\right\|_{V}\right)\left\|v^{m}-v^{0}\right\|_{V} .
$$

Let a sequence $\left\{v^{m}\right\} \subset V$ converge to some limit function $v^{0} \in V$. Then continuity of the map $B_{2}: V \rightarrow X^{*}$ follows from inequality (4.40).

(ii) Let $v \in L_{4}(0, T ; V)$. Quadrating estimate (4.34) and integrating with respect to $t$ from 0 to $T$, we get

$$
\int_{0}^{T}\left\|B_{2}(v)(t)\right\|_{X^{*}}^{2} d t \leqslant C_{7}^{2} \int_{0}^{T}\|v(t)\|_{V}^{4} d t=C_{7}^{2}\|v\|_{L_{4}(0, T ; V)}^{4}
$$

Thus we have that $B_{2}(v) \in L_{2}\left(0, T ; X^{*}\right)$.

Now let us prove continuity of the map $B_{3}: L_{4}(0, T ; V) \rightarrow L_{2}\left(0, T ; X^{*}\right)$.

Let a sequence $\left\{v^{m}\right\} \subset L_{4}(0, T ; V)$ converge to some limit $v^{0} \in L_{4}(0, T ; V)$. We quadrate inequality (4.40) and integrate with respect to $t$ from 0 to $T$. Using the same transformations as above in Lemma 4.5(iii) we get

$$
\begin{aligned}
& \left\|B_{2}\left(v^{m}\right)-B_{2}\left(v^{0}\right)\right\|_{L_{2}\left(0, T ; X^{*}\right)}^{2} \\
& \quad \leq C_{8}^{2}\left(\sum_{i=0}^{4} C_{4}^{i}\left\|v^{m}\right\|_{L_{4}(0, T ; V)}^{i / 4}\left\|v^{0}\right\|_{L_{4}(0, T ; V)}^{(4-i) / 4}\right)^{2}\left\|v^{m}-v^{0}\right\|_{L_{4}(0, T ; V)}^{2} .
\end{aligned}
$$

The continuity of the map $B_{2}: L_{4}(0, T ; V) \rightarrow L_{2}\left(0, T ; X^{*}\right)$ follows from (4.42). 
(iii) Let us use [17, Theorem 2.1, page 184]. We quoted its statement in Lemma 4.1. In this case

$$
\begin{gathered}
X_{0}=X, \quad F=V, \quad X_{1}=L_{2}(\Omega)^{n}, \quad \alpha_{0}=4, \quad \alpha_{1}=2, \\
Y=\left\{v: v \in L_{4}(0, T ; X) ; v^{\prime} \in L_{2}\left(0, T ; L_{2}(\Omega)^{n}\right)\right\} .
\end{gathered}
$$

The embedding $X \subset V$ is compact, therefore the embedding of $Y$ into $L_{4}(0, T ; V)$ is compact.

Since $C([0, T], X) \subset L_{4}(0, T ; X)$ and $L_{2}(0, T ; X) \subset L_{2}\left(0, T ; L_{2}(\Omega)^{n}\right)$ and these embeddings are continuous, $E_{2} \subset Y$ and this embedding is continuous. By (ii) the map $B_{2}$ : $L_{4}(0, T ; V) \rightarrow L_{2}\left(0, T ; X^{*}\right)$ is continuous.

Consequently, we have

$$
E_{2} \subset Y \subset L_{4}(0, T ; V) \stackrel{B_{2}}{\longrightarrow} L_{2}\left(0, T ; X^{*}\right)
$$

Here the first embedding is continuous, the second embedding is compact and the map $B_{2}$ is continuous. Thus for any function $v \in E_{2}$ we have that $B_{2}(v) \in L_{2}\left(0, T ; X^{*}\right)$ and the map $B_{2}: E_{2} \rightarrow L_{2}\left(0, T ; X^{*}\right)$ is compact.

Let us check now estimate (4.35). Quadrating estimate (4.34) and integrating with respect to $t$ from 0 to $T$ we obtain

$$
\int_{0}^{T}\left\|B_{2}(v)(t)\right\|_{X^{*}}^{2} d t \leqslant C_{7}^{2} \int_{0}^{T}\|v(t)\|_{V}^{4} d t \leqslant C_{7}^{2} T\|v\|_{C([0, T], V)}^{4} .
$$

The required estimate (4.35) follows from this inequality.

\section{Existence of weak solution for initial-boundary value problem (3.1)}

Let us introduce operators using the following equalities:

$$
\begin{gathered}
L: E_{2} \longrightarrow L_{2}\left(0, T ; X^{*}\right) \times X, \quad L(v)=\left((J+\varepsilon N+\varkappa A) v^{\prime},\left.v\right|_{t=0}\right), \\
K: E_{2} \longrightarrow L_{2}\left(0, T ; X^{*}\right) \times X, \quad K(v)=\left(v A v-B_{1}(v)-\frac{\varkappa}{2} B_{2}(v)-\frac{\varkappa}{2} B_{3}(v), 0\right) .
\end{gathered}
$$

Then the problem of existence of a solution $v \in E_{2}$ for (4.2), (3.3) is equivalent to the problem of existence of a solution $v \in E_{2}$ for the following operator equation:

$$
L(v)+K(v)=(f, a) .
$$

Together with (5.2) we consider the following family of operator equations:

$$
L(v)+\lambda K(v)=\lambda(f, a), \quad \lambda \in[0,1] .
$$

Observe that (5.3) coincides with (5.2) as $\lambda=1$. 


\subsection{Properties of operators $L$ and $K$}

Lemma 5.1. The operators $L$ and $K$ have the following properties.

(i) The operator $L: E_{2} \rightarrow L_{2}\left(0, T ; X^{*}\right) \times X$ is invertible and the inverse operator $L^{-1}$ : $L_{2}\left(0, T ; X^{*}\right) \times X \rightarrow E_{2}$ is continuous.

(ii) The operator $K: E_{2} \rightarrow L_{2}\left(0, T ; X^{*}\right) \times X$ is completely continuous.

Proof. (i) In order to prove that the operator $L$ is invertible, it suffices to show that the following problem has a unique solution $v \in E_{2}$ for any $f \in L_{2}\left(0, T ; X^{*}\right), a \in X$ :

$$
\begin{gathered}
(J+\varepsilon N+\varkappa A) v^{\prime}=f, \\
\left.v\right|_{t=0}=a .
\end{gathered}
$$

Let us show it.

Since the operator $(J+\varepsilon N+\varkappa A)$ is invertible as an operator from $L_{2}(0, T ; X)$ in $L_{2}(0, T$; $\left.X^{*}\right)$, applying $(J+\varepsilon N+\varkappa A)^{-1}$ to (5.4) we get

$$
v^{\prime}=(J+\varepsilon N+\varkappa A)^{-1} f, \quad v^{\prime} \in L_{2}(0, T ; X) .
$$

Therefore

$$
v(t)=\int_{0}^{t}(J+\varepsilon N+\varkappa A)^{-1} f(s) d s+a, \quad v \in C([0, T], X) .
$$

From (5.6), (5.7) it follows that $v \in E_{2}$. It remains to show that the solution is unique. Really, if there is a second solution $u \in E(u \neq v)$ for problem (5.4), (5.5), the difference $(v-u)$ is a solution for the following problem:

$$
\begin{gathered}
(J+\varepsilon N+\varkappa A)\left(v^{\prime}-u^{\prime}\right)=0 \\
\left.(v-u)\right|_{t=0}=0 .
\end{gathered}
$$

The formula for the solution of this problem, which was obtained above, implies that $u-v=0$. We arrive at a contradiction with the assumption $u \neq v$. Hence, the operator $L$ is invertible.

Let us prove that the inverse operator is continuous. Let a sequence $f_{n}$ converge to some limit $f_{0}$ in $L_{2}\left(0, T ; X^{*}\right)$ and a sequence $a_{n}$ converge to some $a_{0}$ in $X$. Then by formula (5.6) and estimate (4.19) we have

$$
\begin{aligned}
\left\|v_{n}^{\prime}-v_{0}^{\prime}\right\|_{L_{2}(0, T ; X)} & =\left\|(J+\varepsilon N+\varkappa A)^{-1} f_{n}-(J+\varepsilon N+\varkappa A)^{-1} f_{0}\right\|_{L_{2}(0, T ; X)} \\
& \leq C_{3}\left\|f_{n}-f_{0}\right\|_{L_{2}\left(0, T ; X^{*}\right)} .
\end{aligned}
$$

Hence, $v_{n}^{\prime}$ converges to $v_{0}^{\prime}$ in $L_{2}(0, T ; X)$. 
Similarly from (5.7) we get

$$
\begin{aligned}
\| v_{n}- & v_{0} \|_{C([0, T], X)} \\
& =\left\|\int_{0}^{t}(J+\varepsilon N+\varkappa A)^{-1} f_{n}(s) d s+a_{n}-\int_{0}^{t}(J+\varepsilon N+\varkappa A)^{-1} f_{0}(s) d s-a_{0}\right\|_{C([0, T], X)} \\
& \leqslant\left\|\int_{0}^{t}(J+\varepsilon N+\varkappa A)^{-1}\left(f_{n}-f_{0}\right)(s) d s\right\|_{C([0, T], X)}+\left\|a_{n}-a_{0}\right\|_{X} .
\end{aligned}
$$

Using (4.19) and Hölder's inequality we have

$$
\begin{aligned}
\| \int_{0}^{t}(J & +\varepsilon N+\varkappa A)^{-1}\left(f_{n}-f_{0}\right)(s) d s \|_{C([0, T], X)} \\
& =\max _{t \in[0, T]}\left\|\int_{0}^{t}(J+\varepsilon N+\varkappa A)^{-1}\left(f_{n}-f_{0}\right)(s) d s\right\|_{X} \\
& \leq \max _{t \in[0, T]} \int_{0}^{t}\left\|(J+\varepsilon N+\varkappa A)^{-1}\left(f_{n}-f_{0}\right)(s)\right\|_{X} d s \\
& \leq \int_{0}^{T}\left\|(J+\varepsilon N+\varkappa A)^{-1}\left(f_{n}-f_{0}\right)(s)\right\|_{X} d s \\
& \leq C_{3} \int_{0}^{T}\left\|\left(f_{n}-f_{0}\right)(s)\right\|_{X^{*}} d s \\
& \leq C_{3} \sqrt{T}\left(\int_{0}^{T}\left\|\left(f_{n}-f_{0}\right)(s)\right\|_{X^{*}}^{2} d s\right)^{1 / 2}=C_{3} \sqrt{T}\left\|f_{n}-f_{0}\right\|_{L_{2}\left(0, T ; X^{*}\right)} .
\end{aligned}
$$

Thus we have proved that the sequence $v_{n}$ converges to $v_{0}$ in $C([0, T], X)$.

Therefore the sequence $v_{n}$ converges to $v_{0}$ in $E_{2}$. Hence the operator $L^{-1}: L_{2}\left(0, T ; X^{*}\right)$ $\times X \rightarrow E_{2}$ is continuous.

(ii) Compactness of the operator $K: E_{2} \rightarrow L_{2}\left(0, T ; X^{*}\right) \times X$ follows from the compactness of the operators

$$
\begin{array}{ll}
A: E_{2} \longrightarrow L_{2}\left(0, T ; X^{*}\right) & \text { Lemma 4.1(iii), } \\
B_{1}: E_{2} \longrightarrow L_{2}\left(0, T ; X^{*}\right) & \text { Lemma 4.5(iii), } \\
B_{2}: E_{2} \longrightarrow L_{2}\left(0, T ; X^{*}\right) & \text { Lemma 4.6(iii), } \\
B_{3}: E_{2} \longrightarrow L_{2}\left(0, T ; X^{*}\right) & \text { Lemma 4.6(iii). }
\end{array}
$$

\subsection{A priori estimates for solutions of (5.3)}

Theorem 5.2. Let $v \in E_{2}$ be a solution of operator equation (5.3). Then $v$ satisfies the following estimate:

$$
\frac{\varkappa}{2}\|v\|_{C([0, T], V)}^{2}+\varepsilon\|v\|_{C([0, T], X)}^{2} \leqslant C_{9}+\varepsilon\|a\|_{X}^{2} .
$$

Here $C_{9}=(2 T / \varkappa)\|f\|_{L_{2}\left(0, T ; V^{*}\right)}^{2}+\|a\|_{H}^{2}+\varkappa\|a\|_{V}^{2}$. 
Proof. Let $v \in E_{2}$ be a solution of (5.3). Then for any $\varphi \in X$ and almost all $t \in(0, T) v$ satisfies the following equality:

$$
\begin{aligned}
\int_{\Omega} \frac{\partial v}{\partial t} \varphi d x-\lambda \int_{\Omega} v_{i} v_{j} \frac{\partial \varphi_{j}}{\partial x_{i}} d x+\lambda \nu \int_{\Omega} \nabla v: \nabla \varphi d x+\varepsilon \int_{\Omega} \nabla\left(\Delta\left(\frac{\partial v}{\partial t}\right)\right): \nabla(\Delta \varphi) d x \\
\quad+\varkappa \int_{\Omega} \nabla\left(\frac{\partial v}{\partial t}\right): \nabla \varphi d x-\frac{\lambda \varkappa}{2} \int_{\Omega} v_{k} \frac{\partial v_{i}}{\partial x_{j}} \frac{\partial^{2} \varphi_{j}}{\partial x_{i} \partial x_{k}} d x \\
\quad-\frac{\lambda \varkappa}{2} \int_{\Omega} v_{k} \frac{\partial v_{j}}{\partial x_{i}} \frac{\partial^{2} \varphi_{j}}{\partial x_{i} \partial x_{k}} d x=\lambda\langle f, \varphi\rangle .
\end{aligned}
$$

Obviously we have

$$
\begin{aligned}
& \frac{1}{2} \int_{\Omega} v_{k} \frac{\partial v_{i}}{\partial x_{j}} \frac{\partial^{2} \varphi_{j}}{\partial x_{i} \partial x_{k}} d x+\frac{1}{2} \int_{\Omega} v_{k} \frac{\partial v_{j}}{\partial x_{i}} \frac{\partial^{2} \varphi_{j}}{\partial x_{i} \partial x_{k}} d x \\
& \quad=\int_{\Omega} v_{k} \mathscr{E}_{i j}(v) \frac{\partial^{2} \varphi_{j}}{\partial x_{i} \partial x_{k}} d x=-\int_{\Omega} v_{k} \frac{\partial \mathscr{E}_{i j}(v)}{\partial x_{k}} \frac{\partial \varphi_{j}}{\partial x_{i}} d x-\int_{\Omega} \frac{\partial v_{k}}{\partial x_{k}} \mathscr{E}_{i j}(v) \frac{\partial \varphi_{j}}{\partial x_{i}} d x \\
&=-\int_{\Omega} v_{k} \frac{\partial \mathscr{E}_{i j}(v)}{\partial x_{k}} \frac{\partial \varphi_{j}}{\partial x_{i}} d x .
\end{aligned}
$$

Using this equality and (5.14), we get

$$
\begin{gathered}
\int_{\Omega} \frac{\partial v}{\partial t} \varphi d x-\lambda \int_{\Omega} v_{i} v_{j} \frac{\partial \varphi_{j}}{\partial x_{i}} d x+\lambda \nu \int_{\Omega} \nabla v: \nabla \varphi d x+\varepsilon \int_{\Omega} \nabla\left(\Delta\left(\frac{\partial v}{\partial t}\right)\right): \nabla(\Delta \varphi) d x \\
\quad+\varkappa \int_{\Omega} \nabla\left(\frac{\partial v}{\partial t}\right): \nabla \varphi d x+\lambda \varkappa \int_{\Omega} v_{k} \frac{\partial \mathscr{E}_{i j}(v)}{\partial x_{k}} \frac{\partial \varphi_{j}}{\partial x_{i}} d x=\lambda\langle f, \varphi\rangle .
\end{gathered}
$$

Since this equality is valid for all $\varphi \in X$, it is valid for $\varphi=v$. So we have

$$
\begin{gathered}
\int_{\Omega} \frac{\partial v}{\partial t} v d x-\lambda \int_{\Omega} v_{i} v_{j} \frac{\partial v_{j}}{\partial x_{i}} d x+\lambda v \int_{\Omega} \nabla v: \nabla v d x+\varepsilon \int_{\Omega} \nabla\left(\Delta\left(\frac{\partial v}{\partial t}\right)\right): \nabla(\Delta v) d x \\
+\varkappa \int_{\Omega} \nabla\left(\frac{\partial v}{\partial t}\right): \nabla v d x+\lambda \varkappa \int_{\Omega} v_{k} \frac{\partial \mathscr{E}_{i j}(v)}{\partial x_{k}} \frac{\partial v_{j}}{\partial x_{i}} d x=\lambda\langle f, v\rangle .
\end{gathered}
$$

Let us transform the left-hand side of equality (5.17) as follows:

$$
\begin{aligned}
\int_{\Omega} \frac{\partial v}{\partial t} v d x= & \frac{1}{2} \int_{\Omega} \frac{\partial\left(v^{2}\right)}{\partial t} d x=\frac{1}{2} \frac{d}{d t} \int_{\Omega} v^{2} d x=\frac{1}{2} \frac{d}{d t}\|v\|_{H}^{2}, \\
\varepsilon \int_{\Omega} \nabla\left(\Delta\left(\frac{\partial v}{\partial t}\right)\right): \nabla(\Delta v) d x & =\frac{\varepsilon}{2} \int_{\Omega} \frac{\partial}{\partial t}(\nabla(\Delta v): \nabla(\Delta v)) d x=\frac{\varepsilon}{2} \frac{d}{d t} \int_{\Omega} \nabla(\Delta v): \nabla(\Delta v) d x \\
& =\frac{\varepsilon}{2} \frac{d}{d t}\|v\|_{X}^{2},
\end{aligned}
$$




$$
\begin{gathered}
\varkappa \int_{\Omega} \nabla\left(\frac{\partial v}{\partial t}\right): \nabla v d x=\frac{\varkappa}{2} \int_{\Omega} \frac{\partial}{\partial t}(\nabla v: \nabla v) d x=\frac{\varkappa}{2} \frac{d}{d t} \int_{\Omega} \nabla v: \nabla v d x=\frac{\varkappa}{2} \frac{d}{d t}\|v\|_{V}^{2} \\
\int_{\Omega} v_{i} v_{j} \frac{\partial v_{j}}{\partial x_{i}} d x=\int_{\Omega} v_{i} \frac{\partial\left(v_{j} v_{j}\right)}{\partial x_{i}} d x=\int_{\Omega} \frac{\partial v_{i}}{\partial x_{i}} v_{j} v_{j} d x=0 \\
\int_{\Omega} v_{k} \frac{\partial \mathscr{E}_{i j}(v)}{\partial x_{k}} \frac{\partial v_{j}}{\partial x_{i}} d x=2 \int_{\Omega} v_{k} \frac{\partial \mathscr{E}_{i j}(v)}{\partial x_{k}} \mathscr{E}_{i j}(v) d x=2 \int_{\Omega} v_{k} \frac{\partial\left(\mathscr{E}_{i j}(v) \mathscr{E}_{i j}(v)\right)}{\partial x_{k}} d x \\
=-2 \int_{\Omega} \frac{\partial v_{k}}{\partial x_{k}} \mathscr{E}_{i j}(v) \mathscr{E}_{i j}(v) d x=0 .
\end{gathered}
$$

Here we have used the symmetry of the strain rate tensor $\mathscr{E}$.

Thus from (5.17) we obtain

$$
\frac{1}{2} \frac{d}{d t}\|v\|_{H}^{2}+\frac{\varepsilon}{2} \frac{d}{d t}\|v\|_{X}^{2}+\frac{\varkappa}{2} \frac{d}{d t}\|v\|_{V}^{2}+\lambda v\|v\|_{V}^{2}=\lambda\langle f, v\rangle .
$$

Estimating the right-hand side of (5.19) we get

$$
\lambda\langle f, v\rangle \leqslant \lambda\|f\|_{V^{*}}\|v\|_{V} \leqslant\|f\|_{V^{*}}\|v\|_{V}
$$

Estimating the left-hand side of (5.19) we have

$$
\frac{1}{2} \frac{d}{d t}\|v\|_{H}^{2}+\frac{\varepsilon}{2} \frac{d}{d t}\|v\|_{X}^{2}+\frac{\varkappa}{2} \frac{d}{d t}\|v\|_{V}^{2} \leqslant \frac{1}{2} \frac{d}{d t}\|v\|_{H}^{2}+\frac{\varepsilon}{2} \frac{d}{d t}\|v\|_{X}^{2}+\frac{\varkappa}{2} \frac{d}{d t}\|v\|_{V}^{2}+\lambda v\|v\|_{V}^{2} .
$$

Combining (5.20) and (5.21), we obtain

$$
\frac{1}{2} \frac{d}{d t}\|v\|_{H}^{2}+\frac{\varepsilon}{2} \frac{d}{d t}\|v\|_{X}^{2}+\frac{\varkappa}{2} \frac{d}{d t}\|v\|_{V}^{2} \leqslant\|f\|_{V^{*}}\|v\|_{V} .
$$

Integrate this inequality with respect to $t$ from 0 to $\tau$, where $\tau \in[0, T]$ :

$$
\begin{aligned}
& \frac{1}{2}\|v(\tau)\|_{H}^{2}-\frac{\lambda}{2}\|a\|_{H}^{2}+\frac{\varepsilon}{2}\|v(\tau)\|_{X}^{2}-\frac{\varepsilon \lambda}{2}\|a\|_{X}^{2}+\frac{\varkappa}{2}\|v(\tau)\|_{V}^{2}-\frac{\varkappa \lambda}{2}\|a\|_{V}^{2} \\
& \quad \leq \int_{0}^{\tau}\|f(t)\|_{V^{*}}\|v(t)\|_{V} d t .
\end{aligned}
$$

The right-hand side of this inequality may be estimated as follows:

$$
\begin{aligned}
& \int_{0}^{\tau}\|f(t)\|_{V^{*}}\|v(t)\|_{V} d t \\
& \quad \leq \max _{t \in[0, \tau]}\|v(t)\|_{V} \int_{0}^{\tau}\|f(t)\|_{V^{*}} d t \leqslant \max _{t \in[0, T]}\|v(t)\|_{V} \int_{0}^{T}\|f(t)\|_{V^{*}} d t \\
& \quad \leq \sqrt{T} \max _{t \in[0, T]}\|v(t)\|_{V}\left(\int_{0}^{T}\|f(t)\|_{V^{*}}^{2} d t\right)^{1 / 2} \leq \frac{\varkappa}{4}\|v\|_{C([0, T], V)}^{2}+\frac{T}{\varkappa}\|f\|_{L_{2}\left(0, T ; V^{*}\right)}^{2} .
\end{aligned}
$$


Here we used Gölder's inequality and Cauchy's inequality

$$
b c \leqslant \frac{\delta b^{2}}{2}+\frac{c^{2}}{2 \delta}
$$

for $\delta=\varkappa / 2$.

Thus we have

$$
\begin{aligned}
& \frac{1}{2}\|v(\tau)\|_{H}^{2}+\frac{\varepsilon}{2}\|v(\tau)\|_{X}^{2}+\frac{\varkappa}{2}\|v(\tau)\|_{V}^{2} \\
& \quad \leq \frac{T}{\varkappa}\|f\|_{L_{2}\left(0, T ; V^{*}\right)}^{2}+\frac{\varkappa}{4}\|v\|_{C([0, T], V)}^{2}+\frac{1}{2}\|a\|_{H}^{2}+\frac{\varepsilon}{2}\|a\|_{X}^{2}+\frac{\varkappa}{2}\|a\|_{V}^{2} .
\end{aligned}
$$

Since $(1 / 2)\|v(\tau)\|_{H}^{2} \geqslant 0$, we obtain

$$
\frac{\varepsilon}{2}\|v(\tau)\|_{X}^{2}+\frac{\varkappa}{2}\|v(\tau)\|_{V}^{2} \leqslant \frac{T}{\varkappa}\|f\|_{L_{2}\left(0, T ; V^{*}\right)}^{2}+\frac{\varkappa}{4}\|v\|_{C([0, T], V)}^{2}+\frac{1}{2}\|a\|_{H}^{2}+\frac{\varepsilon}{2}\|a\|_{X}^{2}+\frac{\varkappa}{2}\|a\|_{V}^{2} .
$$

The right-hand side of this inequality does not depend from $\tau$. Hence, it is possible to pass to the maxima over $\tau \in[0, T]$ in the left-hand side. So we have

$$
\begin{aligned}
& \frac{\varepsilon}{2}\|v\|_{C([0, T], X)}^{2}+\frac{\varkappa}{2}\|v\|_{C([0, T], V)}^{2} \\
& \quad \leqslant \frac{T}{\varkappa}\|f\|_{L_{2}\left(0, T ; V^{*}\right)}^{2}+\frac{\varkappa}{4}\|v\|_{C([0, T], V)}^{2}+\frac{1}{2}\|a\|_{H}^{2}+\frac{\varepsilon}{2}\|a\|_{X}^{2}+\frac{\varkappa}{2}\|a\|_{V}^{2} .
\end{aligned}
$$

This completes the proof.

Theorem 5.3. Let $v \in E_{2}$ be a solution of (5.3). Then $v$ satisfies the following estimate:

$$
\begin{aligned}
& \varepsilon\left\|v^{\prime}\right\|_{L_{2}(0, T ; X)} \leq C_{10}, \\
& \gamma\left\|v^{\prime}\right\|_{L_{2}\left(0, T ; Z^{*}\right)} \leq 2 C_{10},
\end{aligned}
$$

where

$$
\begin{gathered}
C_{10}=C_{12}+\left(\frac{2 C_{5}}{\varkappa}+2 C_{7} \sqrt{T}\right) \varepsilon\|a\|_{X}^{2}+\nu C_{0} \sqrt{\frac{2\left(C_{9}+\varepsilon\|a\|_{X}^{2}\right)}{\varkappa}}, \\
C_{12}=C_{11}\|f\|_{L_{2}\left(0, T ; V^{*}\right)}+\frac{2 C_{5} C_{9}}{\varkappa}+2 C_{7} C_{9} \sqrt{T} .
\end{gathered}
$$

Proof. Let $v \in E_{2}$ be a solution of (5.2). Then it is a solution for the following operator equation

$$
v^{\prime}-\lambda B_{1}(v)+\lambda \nu A v+\varepsilon N v^{\prime}+\varkappa A v^{\prime}-\frac{\lambda \varkappa}{2} B_{2}(v)-\frac{\lambda \varkappa}{2} B_{3}(v)=\lambda f .
$$

Consequently

$$
\left\|v^{\prime}+\varepsilon N v^{\prime}+\varkappa A v^{\prime}\right\|_{L_{2}\left(0, T ; X^{*}\right)}=\left\|\lambda f+\lambda B_{1}(v)-\lambda v A v+\frac{\lambda \varkappa}{2} B_{2}(v)+\frac{\lambda \varkappa}{2} B_{3}(v)\right\|_{L_{2}\left(0, T ; X^{*}\right)} .
$$


Using (4.4), (4.24), (4.35), (5.13), continuity of the embedding $L_{2}\left(0, T ; V^{*}\right) \subset L_{2}(0, T$; $\left.X^{*}\right)$ and inequality $\lambda \leqslant 1$, we obtain

$$
\begin{aligned}
\| \lambda f+ & \lambda B_{1}(v)-\lambda \nu A v+\frac{\lambda \varkappa}{2} B_{2}(v)+\frac{\lambda \varkappa}{2} B_{3}(v) \|_{L_{2}\left(0, T ; X^{*}\right)} \\
\leq & \lambda\|f\|_{L_{2}\left(0, T ; X^{*}\right)}+\lambda\left\|B_{1}(v)\right\|_{L_{2}\left(0, T ; X^{*}\right)}+\lambda \nu\|A v\|_{L_{2}\left(0, T ; X^{*}\right)} \\
& +\frac{\lambda \varkappa}{2}\left\|B_{2}(v)\right\|_{L_{2}\left(0, T ; X^{*}\right)}+\frac{\lambda \varkappa}{2}\left\|B_{3}(v)\right\|_{L_{2}\left(0, T ; X^{*}\right)} \\
\leq & \|f\|_{L_{2}\left(0, T ; X^{*}\right)}+\left\|B_{1}(v)\right\|_{L_{2}\left(0, T ; X^{*}\right)}+v\|A v\|_{L_{2}\left(0, T ; X^{*}\right)} \\
& +\frac{\varkappa}{2}\left\|B_{2}(v)\right\|_{L_{2}\left(0, T ; X^{*}\right)}+\frac{\varkappa}{2}\left\|B_{3}(v)\right\|_{L_{2}\left(0, T ; X^{*}\right)} \\
\leq & C_{11}\|f\|_{L_{2}\left(0, T ; V^{*}\right)}+C_{5}\|v\|_{C([0, T], V)}^{2}+\nu C_{0}\|v\|_{C([0, T], V)}+\varkappa C_{7} \sqrt{T}\|v\|_{C([0, T], V)}^{2} \\
\leq & C_{11}\|f\|_{L_{2}\left(0, T ; V^{*}\right)}+\frac{2 C_{5}\left(C_{9}+\varepsilon\|a\|_{X}^{2}\right)}{\varkappa}+\nu C_{0} \sqrt{\frac{2\left(C_{9}+\varepsilon\|a\|_{X}^{2}\right)}{\varkappa}} \\
& +2 C_{7}\left(C_{9}+\varepsilon\|a\|_{X}^{2}\right) \sqrt{T .}
\end{aligned}
$$

Combining this inequality and (4.12) we get the inequality (5.29).

Similarly we have

$$
\begin{aligned}
\left\|v^{\prime}+\varkappa A v^{\prime}\right\|_{L_{2}\left(0, T ; X^{*}\right)}= & \left\|\varepsilon N v^{\prime}+\lambda f+\lambda B_{1}(v)-\lambda \nu A v+\frac{\lambda \varkappa}{2} B_{2}(v)+\frac{\lambda \varkappa}{2} B_{3}(v)\right\|_{L_{2}\left(0, T ; X^{*}\right)} \\
\leq & \left\|\varepsilon N v^{\prime}\right\|_{L_{2}\left(0, T ; X^{*}\right)} \\
& +\left\|\lambda f+\lambda B_{1}(v)-\lambda v A v+\frac{\lambda \varkappa}{2} B_{2}(v)+\frac{\lambda \varkappa}{2} B_{3}(v)\right\|_{L_{2}\left(0, T ; X^{*}\right)} \\
\leq & \varepsilon\left\|v^{\prime}\right\|_{L_{2}(0, T ; X)}+C_{10} \leq 2 C_{10} .
\end{aligned}
$$

Further the operator $(I-\varkappa \Delta): L_{2}(0, T ; X) \rightarrow L_{2}(0, T ; Z)$ is invertible because $\operatorname{ker}(I-$ $\varkappa \Delta)=\{0\}$ and the image of $L_{2}(0, T ; X)$ under the action of $(I-\varkappa \Delta)$ coincides with $L_{2}(0, T ; Z)$. The adjont operator to $(I-\varkappa \Delta): L_{2}(0, T ; X) \rightarrow L_{2}(0, T ; Z)$ is the operator $(I-\varkappa \Delta)^{*}: L_{2}\left(0, T ; Z^{*}\right) \rightarrow L_{2}\left(0, T ; X^{*}\right)$. But we have that $(I-\varkappa \Delta)^{*}=(I-\varkappa \Delta)$.

Then the operator $(I-\varkappa \Delta): L_{2}\left(0, T ; Z^{*}\right) \rightarrow L_{2}\left(0, T ; X^{*}\right)$ is invertible. Hence there exists $\gamma>0$ such that

$$
\|(I-\varkappa \Delta) u\|_{L_{2}\left(0, T ; X^{*}\right)} \geq \gamma\|u\|_{L_{2}\left(0, T ; Z^{*}\right)}
$$

From this inequality we have estimate (5.30).

Theorems 5.2 and 5.3 imply the following corollary. 
Corollary 5.4. Let $v \in E_{2}$ be a solution of the operator equation (5.3). Then the following estimates take place:

$$
\begin{aligned}
\|v\|_{E_{2}} \leq & C_{13}=\sqrt{\frac{C_{9}}{\varepsilon}+\|a\|_{X}^{2}}+\frac{C_{10}}{\varepsilon}, \\
\|v\|_{E_{1}} \leq & C_{14}=\frac{2 C_{12}}{\gamma}+\sqrt{\frac{2\left(C_{9}+\varepsilon\|a\|_{X}^{2}\right)}{\varkappa}} \\
& +\frac{\left(4 C_{5} / \varkappa+4 C_{7} \sqrt{T}\right) \varepsilon\|a\|_{X}^{2}+2 \nu C_{0} \sqrt{2\left(C_{9}+\varepsilon\|a\|_{X}^{2}\right) / \varkappa}}{\gamma} .
\end{aligned}
$$

\subsection{Existence theorem for solutions of operator equation (5.2)}

Theorem 5.5. The operator equation (5.2) has at least one solution $v \in E_{2}$.

Proof. In the proof of this theorem we use the Leray-Schauder degree theory [10].

Consider the following family of operator equations

$$
L(v)+\lambda K(v)=\lambda(f, a), \quad \lambda \in[0,1] .
$$

By Corollary 5.4 all solutions of this family of operator equations satisfy a priori estimate (5.37). Therefore all equations of this family have no solutions on the boundary of the ball $B_{R} \subset E_{2}$ of radius $R=C_{13}+1$ with center at zero. Consequently all equations of the family of operator equations

$$
v+\lambda L^{-1}[K(v)-(f, a)]=0, \quad \lambda \in[0,1],
$$

have no solutions on the boundary of the same ball $B_{R} \subset E_{2}$. By Lemma 5.1(ii) the map

$$
[K(\cdot)-(f, a)]: E_{2} \longrightarrow L_{2}\left(0, T ; X^{*}\right) \times X
$$

is compact. By Lemma 5.1(i) the operator

$$
L^{-1}: L_{2}\left(0, T ; X^{*}\right) \times X \longrightarrow E_{2}
$$

is continuous.

Therefore the map

$$
L^{-1}[K(\cdot)-(f, a)]: E_{2} \longrightarrow E_{2}
$$

is compact. Let us introduce the map

$$
G:[0,1] \times E_{2} \longrightarrow E_{2}, \quad G(\lambda, v)=\lambda L^{-1}[K(v)-(f, a)] .
$$

By the above reasonings this map is compact on the set of variables $\lambda$ and $\nu$.

Thus we have that the compact vector field

$$
\Phi(\lambda, v)=v-G(\lambda, v)
$$


is non-degenerate on the boundary of the ball $B_{R}$. Consequently the topological degree $\operatorname{deg}_{\mathrm{LS}}\left(\Phi, B_{R}, 0\right)$ is well defined.

Using the property of homotopic invariance of the degree we get

$$
\operatorname{deg}_{\mathrm{LS}}\left(\Phi(0, \cdot), B_{R}, 0\right)=\operatorname{deg}_{\mathrm{LS}}\left(\Phi(1, \cdot), B_{R}, 0\right) .
$$

We recall that $\Phi(0, \cdot)=I$ and $\operatorname{deg}_{\mathrm{LS}}\left(I, B_{R}, 0\right)=1$. This implies that

$$
\operatorname{deg}_{\mathrm{LS}}\left(\Phi(1, \cdot), B_{R}, 0\right)=1
$$

Since this degree is nonzero, there exists at least one solution $v \in E_{2}$ of the operator equation

$$
v+L^{-1}[K(v)-(f, a)]=0 .
$$

Hence there exists at least one solution $v \in E_{2}$ of the operator equation (5.2). This completes the proof.

Since there is at least one solution $v \in E_{2}$ of (5.2), from the above reasonings it follows that the initial-boundary value problem (3.1) has at least one weak solution $v \in E_{2}$.

\section{Proof of Theorem 3.3}

Since $X$ is dense in $V$, there exists a sequence $a_{m} \in X$ converging to $a$ in $V$. If $a \equiv 0$ we put

$$
a_{m} \equiv 0, \quad \varepsilon_{m}=\frac{1}{m} .
$$

If $\|a\|_{V} \neq 0$, there exists some number $m_{0}$ such that $\left\|a_{m}\right\|_{X} \neq 0$ for $m>m_{0}$. Then we put

$$
\varepsilon_{m}=\frac{1}{m\left\|a_{m}\right\|_{X}^{2}} .
$$

The sequence $\left\{\varepsilon_{m}\right\}$ converges to zero as $m \rightarrow+\infty$. By Theorem 5.5 for each $\varepsilon_{m}$ and $a_{m}$ there exists a weak solution $v_{m} \in E_{2} \subset E_{1}$ for the initial-boundary value problem (3.1). Then every $v_{m}$ satisfies equality (3.2):

$$
\begin{aligned}
& \int_{\Omega} \frac{\partial v_{m}}{\partial t} \varphi d x-\int_{\Omega}\left(v_{m}\right)_{i}\left(v_{m}\right)_{j} \frac{\partial \varphi_{j}}{\partial x_{i}} d x+\nu \int_{\Omega} \nabla v_{m}: \nabla \varphi d x+\varepsilon_{m} \int_{\Omega} \nabla\left(\Delta\left(\frac{\partial v_{m}}{\partial t}\right)\right): \nabla(\Delta \varphi) d x \\
& \quad+\varkappa \int_{\Omega} \nabla\left(\frac{\partial v_{m}}{\partial t}\right): \nabla \varphi d x-\frac{\varkappa}{2} \int_{\Omega}\left(v_{m}\right)_{k} \frac{\partial\left(v_{m}\right)_{i}}{\partial x_{j}} \frac{\partial^{2} \varphi_{j}}{\partial x_{i} \partial x_{k}} d x \\
& \quad-\frac{\varkappa}{2} \int_{\Omega}\left(v_{m}\right)_{k} \frac{\partial\left(v_{m}\right)_{j}}{\partial x_{i}} \frac{\partial^{2} \varphi_{j}}{\partial x_{i} \partial x_{k}} d x=\langle f, \varphi\rangle .
\end{aligned}
$$

and initial condition (3.3):

$$
\left.v_{m}\right|_{t=0}(x)=a_{m}(x), \quad x \in \Omega .
$$


Using the continuous embedding $C([0, T], V) \subset L_{4}(0, T ; V)$ and estimates (5.13) and (5.30) without loss of generality (and passing to a subsequence if necessary), we get

$$
\begin{aligned}
& v_{m} \longrightarrow v_{*} \text {, weakly in } L_{4}(0, T ; V) \text {; } \\
& v_{m}^{\prime} \longrightarrow v_{*}^{\prime} \text {, weakly in } L_{2}\left(0, T ; Z^{*}\right) \text {. }
\end{aligned}
$$

Hence by definition of weak convergence we obtain

$$
\begin{gathered}
\nu \int_{\Omega} \nabla v_{m}: \nabla \varphi d x \longrightarrow v \int_{\Omega} \nabla v_{*}: \nabla \varphi d x \\
\int_{\Omega} \frac{\partial v_{m}}{\partial t} \varphi d x+\varkappa \int_{\Omega} \nabla\left(\frac{\partial v_{m}}{\partial t}\right): \nabla \varphi d x=\left\langle(I-\varkappa \Delta) \frac{\partial v_{m}}{\partial t}, \varphi\right\rangle \longrightarrow\left\langle(I-\varkappa \Delta) \frac{\partial v_{*}}{\partial t}, \varphi\right\rangle
\end{gathered}
$$

as $m \rightarrow \infty$.

Using estimate (5.29) as above without loss of generality we have that there exists $u \in$ $L_{2}(0, T ; X)$ such that

$$
\varepsilon_{m} v_{m}^{\prime} \longrightarrow u, \quad \text { weakly in } L_{2}(0, T ; X) \text {. }
$$

However, in the sense of distributions the sequence $\varepsilon_{m} v_{m}^{\prime}$ converges to zero. Really, for any $\chi \in \mathfrak{D}([0, T]), \varphi \in \mathfrak{D}(\Omega)^{n}$ we have

$$
\begin{aligned}
& \lim _{m \rightarrow \infty}\left|\int_{0}^{T} \int_{\Omega} \nabla\left(\Delta\left(\frac{\partial\left(\varepsilon_{m} v_{m}\right)}{\partial t}(t)\right)\right): \nabla(\Delta \varphi) d x \chi(t) d t\right| \\
& =\lim _{m \rightarrow \infty} \varepsilon_{m}\left|\int_{0}^{T} \int_{\Omega} \nabla\left(\Delta\left(\frac{\partial v_{m}}{\partial t}(t)\right)\right): \nabla(\Delta \varphi) d x \chi(t) d t\right| \\
& =\lim _{m \rightarrow \infty} \varepsilon_{m}\left|\int_{0}^{T} \int_{\Omega} \Delta\left(\frac{\partial v_{m}}{\partial t}(t)\right) \Delta^{2} \varphi d x \chi(t) d t\right| \\
& =\lim _{m \rightarrow \infty} \varepsilon_{m}\left|\int_{0}^{T} \int_{\Omega} \nabla\left(\frac{\partial v_{m}}{\partial t}(t)\right): \nabla\left(\Delta^{2} \varphi\right) d x \chi(t) d t\right| \\
& =\lim _{m \rightarrow \infty} \varepsilon_{m} \lim _{m \rightarrow \infty}\left|\int_{0}^{T} \int_{\Omega} \nabla\left(\frac{\partial v_{m}}{\partial t}(t)\right): \nabla\left(\Delta^{2} \varphi\right) d x \chi(t) d t\right| \\
& =\lim _{m \rightarrow \infty} \varepsilon_{m} \lim _{m \rightarrow \infty}\left|\int_{\Omega}\left(\int_{0}^{T} \nabla\left(\frac{\partial v_{m}}{\partial t}(t)\right) \chi(t) d t\right): \nabla\left(\Delta^{2} \varphi\right) d x\right| \\
& =\lim _{m \rightarrow \infty} \varepsilon_{m} \lim _{m \rightarrow \infty}\left|\int_{\Omega}\left(\int_{0}^{T} \nabla v_{m}(t) \frac{\partial \chi(t)}{\partial t} d t\right): \nabla\left(\Delta^{2} \varphi\right) d x\right| \\
& =\lim _{m \rightarrow \infty} \varepsilon_{m} \lim _{m \rightarrow \infty}\left|\int_{0}^{T} \int_{\Omega} \nabla v_{m}(t): \nabla\left(\Delta^{2} \varphi\right) d x \frac{\partial \chi(t)}{\partial t} d t\right| \\
& =\left|\int_{0}^{T} \int_{\Omega} \nabla v_{*}(t): \nabla\left(\Delta^{2} \varphi\right) d x \frac{\partial \chi(t)}{\partial t} d t\right| \lim _{m \rightarrow \infty} \varepsilon_{m}=0 .
\end{aligned}
$$


Here we have used the fact that if the sequence $v_{m}^{\prime}$ converges weakly to $v_{*}^{\prime}$ in $L_{2}(0, T ; V)$ then it converges to $v_{*}^{\prime}$ in the sense of distributions.

Thus by uniqueness of a weak limit $\varepsilon_{m} v_{m}^{\prime} \rightarrow 0$ weakly in $L_{2}(0, T ; X)$ as $m \rightarrow+\infty$. Hence

$$
\varepsilon_{m} \int_{\Omega} \nabla\left(\Delta\left(\frac{\partial v_{m}}{\partial t}\right)\right): \nabla(\Delta \varphi) d x \longrightarrow 0 \quad \text { as } m \longrightarrow+\infty .
$$

By the same arguments as in Lemma 4.5(iii) we obtain the compact embedding

$$
Y=\left\{v: v \in L_{4}(0, T ; V) ; v^{\prime} \in L_{2}\left(0, T ; Z^{*}\right)\right\} \subset L_{4}\left(0, T ; L_{4}(\Omega)^{n}\right) .
$$

Combining this compact embedding and estimate (5.38) we get

$$
v_{m} \longrightarrow v_{*} \quad \text { strongly in } L_{4}\left(0, T ; L_{4}(\Omega)^{n}\right) .
$$

This implies that

$$
\int_{\Omega}\left(v_{m}\right)_{i}\left(v_{m}\right)_{j} \frac{\partial \varphi_{j}}{\partial x_{i}} d x \longrightarrow \int_{\Omega}\left(v_{*}\right)_{i}\left(v_{*}\right)_{j} \frac{\partial \varphi_{j}}{\partial x_{i}} d x .
$$

Furthermore we obtain

$$
\begin{aligned}
& \frac{\varkappa}{2} \int_{\Omega}\left(v_{m}\right)_{k} \frac{\partial\left(v_{m}\right)_{i}}{\partial x_{j}} \frac{\partial^{2} \varphi_{j}}{\partial x_{i} \partial x_{k}} d x \rightarrow \frac{\varkappa}{2} \int_{\Omega}\left(v_{*}\right)_{k} \frac{\partial\left(v_{*}\right)_{i}}{\partial x_{j}} \frac{\partial^{2} \varphi_{j}}{\partial x_{i} \partial x_{k}} d x, \\
& \frac{\varkappa}{2} \int_{\Omega}\left(v_{m}\right)_{k} \frac{\partial\left(v_{m}\right)_{j}}{\partial x_{i}} \frac{\partial^{2} \varphi_{j}}{\partial x_{i} \partial x_{k}} d x \rightarrow \frac{\varkappa}{2} \int_{\Omega}\left(v_{*}\right)_{k} \frac{\partial\left(v_{*}\right)_{j}}{\partial x_{i}} \frac{\partial^{2} \varphi_{j}}{\partial x_{i} \partial x_{k}} d x .
\end{aligned}
$$

Indeed, the sequence $v_{m}$ converges to $v_{*}$ strongly in $L_{4}\left(0, T ; L_{4}(\Omega)^{n}\right)$ and the sequence $\nabla\left(v_{m}\right)$ converges to $\nabla v_{*}$ weakly in $L_{4}\left(0, T ; L_{2}(\Omega)^{n}\right)$. This yields that their product $v_{m} \nabla v_{m}$ converges to $v_{*} \nabla v_{*}$ weakly in $L_{2}\left(0, T ; L_{4 / 3}(\Omega)^{n}\right)$.

Thus passing in each term of equality (6.3) to the limit as $m \rightarrow+\infty$, we obtain that the limit function $v_{*}$ satisfies the following equality:

$$
\begin{aligned}
& \left\langle(I-\varkappa \Delta) \frac{\partial v_{*}}{\partial t}, \varphi\right\rangle-\int_{\Omega}\left(v_{*}\right)_{i}\left(v_{*}\right)_{j} \frac{\partial \varphi_{j}}{\partial x_{i}} d x+v \int_{\Omega} \nabla v_{*}: \nabla \varphi d x \\
& \quad-\frac{\varkappa}{2} \int_{\Omega}\left(v_{*}\right)_{k} \frac{\partial\left(v_{*}\right)_{i}}{\partial x_{j}} \frac{\partial^{2} \varphi_{j}}{\partial x_{i} \partial x_{k}} d x-\frac{\varkappa}{2} \int_{\Omega}\left(v_{*}\right)_{k} \frac{\partial\left(v_{*}\right)_{j}}{\partial x_{i}} \frac{\partial^{2} \varphi_{j}}{\partial x_{i} \partial x_{k}} d x=\langle f, \varphi\rangle .
\end{aligned}
$$

Passing in initial condition (6.4) to the limit as $m \rightarrow+\infty$, we obtain that $v_{*}$ satisfies the following initial condition:

$$
\left.v_{*}\right|_{t=0}(x)=a(x), \quad x \in \Omega .
$$

Since for $\left\{v_{m}\right\}$ a priori estimate (5.38) is valid, for $v_{*}$ the following estimate takes place

$$
\left\|v_{*}\right\|_{L_{\infty}(0, T ; V)}+\left\|v_{*}^{\prime}\right\|_{L_{2}\left(0, T ; Z^{*}\right)} \leq C_{15}
$$


Here $C_{15}=2 C_{12} / \gamma+\sqrt{2 C_{9} \varkappa}+\left(2 \nu C_{0} \sqrt{2 C_{9} / \varkappa}\right) / \gamma$. We obtain this estimate by passing to the limit in estimate (5.38).

Therefore we have that $v_{*} \in E_{1}$ and it satisfies the estimate:

$$
\left\|v_{*}\right\|_{E_{1}} \leqslant C_{15}
$$

This completes the proof.

\section{Acknowledgment}

The work was partially supported by Grants 04-01-00081 of Russian Foundation of Basic Research and VZ-010-0 of Ministry of Education of Russia and CRDF.

\section{References}

[1] V. B. Amfilohiev and V. A. Pavlovsky, Experimental data about laminar-turbulent passage at flow of a polymer solutions in pipes, Trudy Leningradskogo korablestroitelnogo Instituta 104 (1976), 3-5 (Russian).

[2] V. B. Amfilohiev, Ya. I. Vojtkunsky, N. P. Mazaeva, and Ya. S. Khodorkovsky, The flow of a polymer solutions at presence of convective accelerations, Trudy Leningradskogo korablestroitelnogo Instituta 96 (1975), 3-9 (Russian).

[3] A. M. Freudental and H. Geiringer, The mathematical theories of the inelastic continuum, Handbuch der Physik, herausgegeben von S. Flügge. Bd. 6. Elastizität und Plastizität, Springer, Berlin, 1958, pp. 229-433.

[4] H. Gajewski, K. Gröger, and K. Zacharias, Nichtlineare Operatorgleichungen und Operatordifferentialgleichungen, Mathematische Lehrbücher und Monographien, II. Abteilung, Mathematische Monographien, vol. 38, Akademie, Berlin, 1974.

[5] O. A. Ladyzhenskaya, On the nonstationary Navier-Stokes equations, Vestnik Leningrad University. Mathematics 13 (1958), no. 19, 9-18 (Russian).

[6] - The Mathematical Theory of Viscous Incompressible Flow, Fizmatgiz, Moscow, 1961.

[7] _ On errors in two of my publications on Navier-Stokes equations and their corrections, Rossiŭ skaya Akademiya Nauk. Sankt-Peterburgskoe Otdelenie. Matematicheskiŭ Institut im. V. A. Steklova. Zapiski Nauchnykh Seminarov (POMI) 271 (2000), 151-155, 316 (Russian).

[8] J.-L. Lions, Quelques méthodes de résolution des problèmes aux limites non linéaires, Dunod; Gauthier-Villars, Paris, 1969.

[9] V. G. Lìtvīnov, Motion of a Nonlinearly Viscous Fluid, Nauka, Moscow, 1982.

[10] N. G. Lloyd, Degree Theory, Cambridge Tracts in Mathematics, no. 73, Cambridge University Press, Cambridge, 1978.

[11] A. P. Oskolkov, Solvability in the large of the first boundary value problem for a certain quasilinear third order system that is encountered in the study of the motion of a viscous fluid, Zapiski Naučnyh Seminarov Leningradskogo Otdelenija Matematičeskogo Instituta im. V. A. Steklova Akademii Nauk SSSR (LOMI) 27 (1972), 145-160 (Russian).

[12] The uniqueness and solvability in the large of boundary value problems for the equations of motion of aqueous solutions of polymers, Zapiski Naučnyh Seminarov Leningradskogo Otdelenija Matematičeskogo Instituta im. V. A. Steklova Akademii Nauk SSSR (LOMI) 38 (1973), 98-136 (Russian).

[13] Some quasilinear systems that arise in the study of the motion of viscous fluids, Zapiski Naučnyh Seminarov Leningradskogo Otdelenija Matematičeskogo Instituta im. V. A. Steklova Akademii Nauk SSSR (LOMI) 52 (1975), 128-157, 219. 
[14]_ Some nonstationary linear and quasilinear systems that arise in the study of the motion of viscous fluids, Zapiski Naučnyh Seminarov Leningradskogo Otdelenija Matematičeskogo Instituta im. V. A. Steklova Akademii Nauk SSSR (LOMI) 59 (1976), 133-177, 257 (Russian).

[15] __ Initial-boundary value problems with a sliding boundary condition for modified NavierStokes equations, Rossiǐ skaya Akademiya Nauk. Sankt-Peterburgskoe Otdelenie. Matematicheskiĭ Institut im. V. A. Steklova. Zapiski Nauchnykh Seminarov (POMI) 213 (1994), 93-115, 225-226 (Russian).

[16] V. A. Pavlovsky, To a problem on theoretical exposition of weak aqueous solutions of polymers, Doklady Akademii Nauk SSSR 200 (1971), no. 4, 809-812 (Russian).

[17] R. Temam, Navier-Stokes Equations: Theory and Numerical Analysis, AMS Chelsea, Rhode Island, 2001.

[18] Ya. I. Vojtkunsky, V. B. Amfilohiev, and V. A. Pavlovsky, Equations of motion of a liquid with taking into account its relaxational properties, Trudy Leningradskogo korablestroitelnogo Instituta 69 (1970), 19-26 (Russian).

[19] V. G. Zvyagin and V. T. Dmitrienko, The Approximating Approach to Research of Problems of Fluid Dynamics. The Navier-Stokes System, Editorial URSS, Moscow, 2004.

Mikhail V. Turbin: Voronezh State University, Universitetskaya pl.1, 394006 Voronezh, Russia

E-mail address: mrmike@math.vsu.ru 


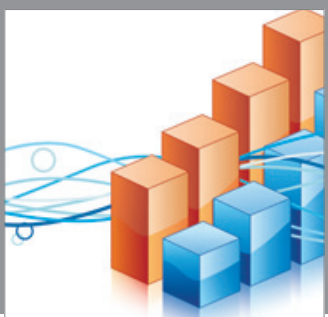

Advances in

Operations Research

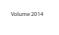

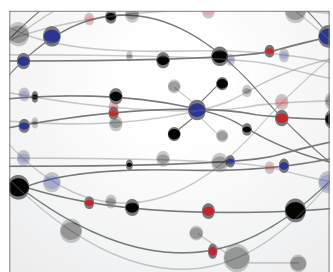

\section{The Scientific} World Journal
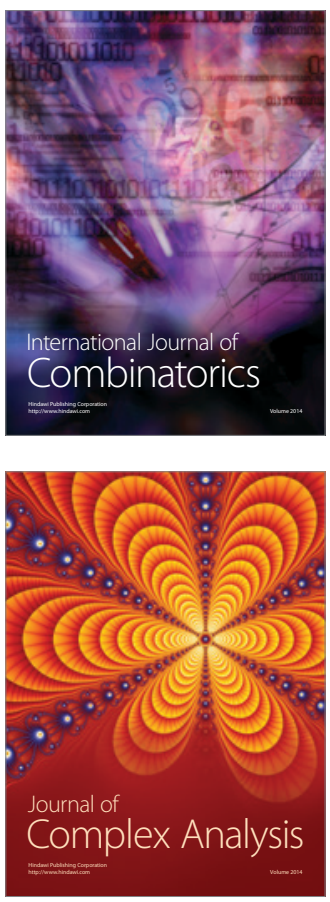

International Journal of

Mathematics and

Mathematical

Sciences
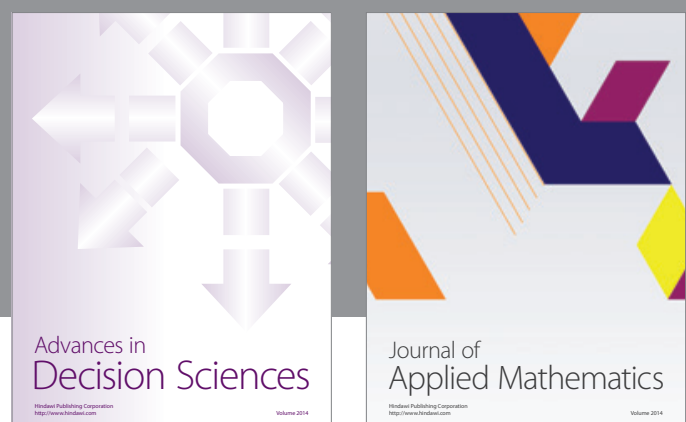

Journal of

Applied Mathematics
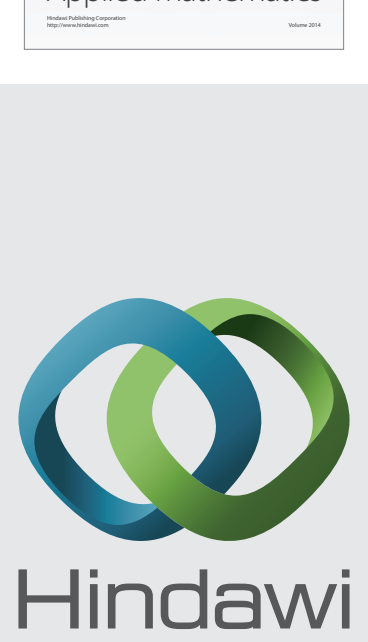

Submit your manuscripts at http://www.hindawi.com
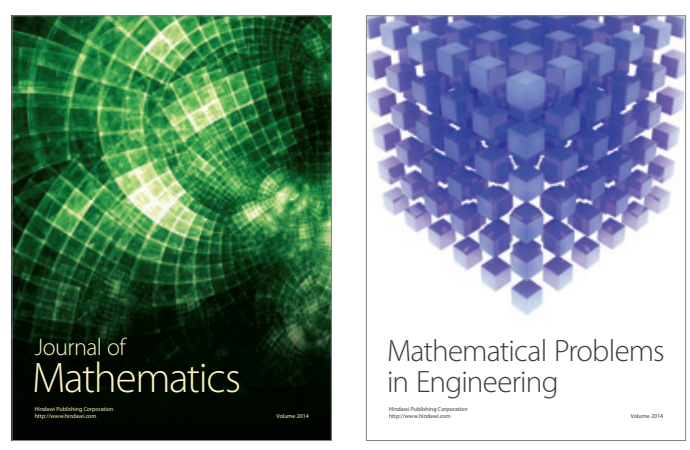

Mathematical Problems in Engineering
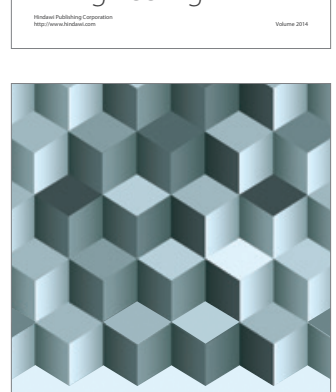

Journal of

Function Spaces
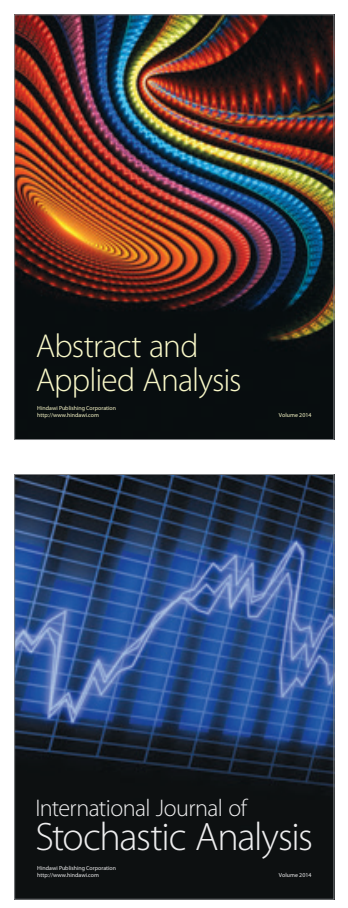

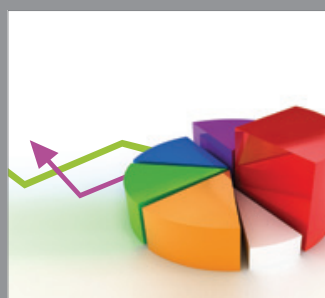

ournal of

Probability and Statistics

Promensencen
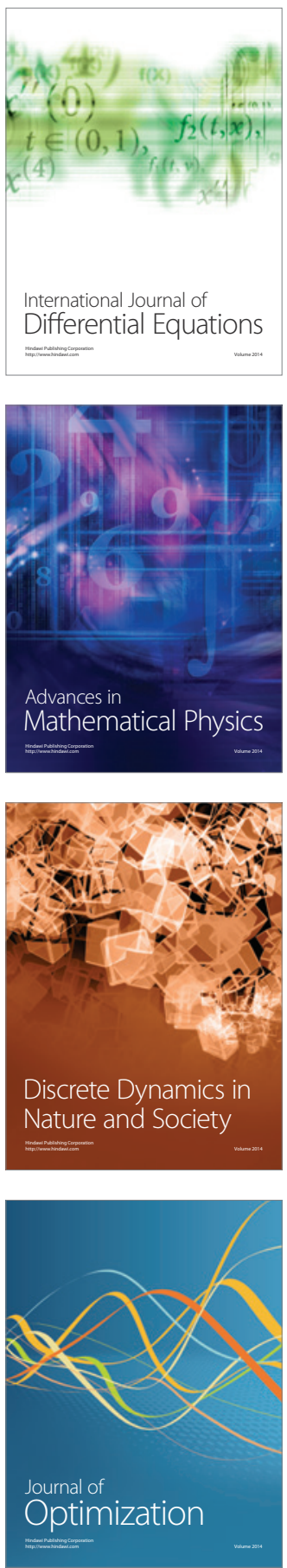